Research Article

\title{
Effective Material Basis and Mechanism Analysis of Compound Banmao Capsule against Tumors Using Integrative Network Pharmacology and Molecular Docking
}

\author{
Tian-Mu He $\mathbb{D}^{1},{ }^{1}$ Jing-Xian Liu $\mathbb{D}^{1},{ }^{1}$ Can-Can Duan $\mathbb{D}^{2,3}$ Xiao-Fei Li ${ }^{(\mathbb{D})},{ }^{1,2}$ \\ and Jian-Yong Zhang $\mathbb{C}^{2,3}$ \\ ${ }^{1}$ Basic Medicine School, Zunyi Medical University, Zunyi, China \\ ${ }^{2}$ School of Pharmacy, Zunyi Medical University, Zunyi, China \\ ${ }^{3}$ Key Laboratory of Basic Pharmacology Ministry Education and Joint International Research Laboratory of Ethnomedicine \\ Ministry of Education, Zunyi Medical University, Zunyi, China
}

Correspondence should be addressed to Xiao-Fei Li; lixiaofei@zmu.edu.cn and Jian-Yong Zhang; zhangjianyong2006@126.com

Received 7 November 2020; Revised 24 March 2021; Accepted 26 March 2021; Published 4 May 2021

Academic Editor: Amin Tamadon

Copyright $@ 2021$ Tian-Mu He et al. This is an open access article distributed under the Creative Commons Attribution License, which permits unrestricted use, distribution, and reproduction in any medium, provided the original work is properly cited.

Purpose. Compound banmao capsule (CBC), a well-known traditional Chinese medical material, is known to inhibit various tumors. However, its material basis and pharmacological mechanisms remain to be elucidated. This study aimed to investigate the effective material basis and mechanisms of action of CBC against tumors. Methods. Active compounds of CBC were identified using public database and reports to build a network. The corresponding targets of active compounds were retrieved from online databases, and the antitumor targets were identified by GeneCards database. The antitumor hub targets were generated via protein-protein interaction analysis using String, and key compounds and targets from the integrative network were detected by molecular docking and ADMET. Top targets in hepatocellular carcinoma were confirmed by quantitative real-time PCR (qPCR). Finally, the multivariate biological network was built to identify the integrating mechanisms of action of CBC against tumor cells. Results. A total of 128 compounds and 436 targets of CBC were identified successfully. Based on the generated multivariate biological network analysis, 25 key compounds, nine hub targets, and two pathways were further explored. Effective material bases of cantharidin, baicalein, scutellarin, sesamin, and quercetin were verified by integrative network analysis. PTGS2, ESR1, and TP53 were identified as hub targets via multivariate biological network analysis and confirmed using qPCR. Furthermore, VEGF and estrogen signaling pathways seem to play a role in the antitumor activity of CBC. Thus, breast cancer may be a potential clinical indication of CBC. Conclusion. This study successfully identified the material basis of CBC and its synergistic mechanisms of action against tumor cells.

\section{Introduction}

Studies have shown that the five-year survival rate of cancer generally rise when tumor survival trends in global areas are evaluated. However, the survival rate of malignant tumors, such as liver cancer, is still low [1]. At present, antitumor therapy is mainly based on radiotherapy and chemotherapy, which are linked to severe adverse reactions [2]. Thus, exploring new antitumor drugs with fewer side effects is urgently needed. Traditional Chinese medicine (TCM) is popular, plays a role in the modern healthcare system, and can provide potential effective strategies towards the treatment of tumors [3]. Compared with conventional drugs, herbal drugs have been considered an important supplementary treatment to alleviate the side effects of both radiotherapy and chemotherapy [4].

Compound banmao capsule (CBC) is one of the most common antitumor TCM compounds composed of 11 herbs according to the Pharmacopoeia of the People's Republic of China (2020 edition), including the Mylabris (BM), Ginseng Radix Et Rhizoma (RS), Astragali Radix (HQ), Scutellariae Barbatae Herba (BZL), Curcumae Rhizoma (EZ), Acanthopanacis Senticosi Radix Et Rhizoma Seu Caulis (CWJ), 
Fel Ursi (XD), Glycyrrhizae Radix Et Rhizoma (GC), Corni Fructus (SZY), Ligustri Lucidi Fructus (NZZ), and Sparganii Rhizoma (SL). CBC has been used to clinically treat primary liver tumor, lung tumor, rectal tumor, and malignant lymphoma [5]. Most notably, it differs from other antitumor drugs as CBC not only can directly inhibit tumor proliferation but also enhance immunity of patients. Modern antitumor mechanisms of CBC mainly involve proapoptosis, protein phosphorylation, and immunoregulation [6-8]. However, the specific pharmacodynamic material and molecular mechanisms underlying tumor treatment of CBC still remain unclear.

Regarding the integrative network pharmacology, a multicompound, multitarget, and multipathway approach has been used in TCM, especially in antitumor research $[9,10]$. Here, we attempt to identify effective material basis and molecular mechanisms of CBC using network pharmacology and molecular docking. Hub targets aim to be verified by quantitative real-time PCR (qPCR) in human hepatocellular carcinoma cells (SMMC-7721 cell), providing a theoretical basis for further research.

\section{Materials and Methods}

\subsection{Network Pharmacology-Based Analysis}

2.1.1. Identification of Candidate Compounds of CBC. All compounds of $11 \mathrm{TCM}$ herbs of CBC were retrieved from the encyclopedia of TCM (ETCM) database (http://www. nrc.ac.cn:9090/ETCM/) and literature reports. ETCM is an encyclopedia of TCM, which includes comprehensive and standardized information for the commonly used herbs and formulas of TCM as well as their ingredients [11].

2.1.2. Screening for Bioactive Compounds of $C B C$. A quantitative metrics, known as the quantitative estimate of drug likeness (QED), was used to assess drug likeness. Estimated values ranged from 0 (all properties are unfavorable) to 1 (all properties are favorable) [12]. ETCM classified all 7274 ingredients into three groups according to their QED scores: good (QED > 0.67), moderate $(0.49 \leq \mathrm{QED} \leq 0.67)$, and weak $(\mathrm{QED}<0.49)$ [11]. Compounds of CBC evaluated as good $(\mathrm{QED}>0.67)$ were collected as bioactive compounds. Likewise, active compounds that did not fulfill the criteria for QED but have been reported in the available literature were recruited separately.

\subsubsection{Prediction of Drug Targets for $C B C$ and Network} Construction. Targets of active compounds of CBC were retrieved from ETCM. Other active compounds not included in ETCM were drawn via ChemDraw 19.0 software and imported into TargetNet database (http://targetnet. scbdd.com/), a web service for predicting potential drugtarget interaction profiling via multitarget SAR models, for predicting targets [13]. Targets were filtered by accuracy (AUC) $\geq 0.7$ and probability (Prob) $\geq 0.9$. Targets were corrected and transformed using UniProt database (https:// www.uniprot.org/) and defined as Homo sapiens. Subsequently, correction targets were imported into Cytoscape 3.7.0 software to construct a network of herb-compoundstargets.

2.1.4. Collection of Gene Targets Associated with Tumors. The terms "anti-tumor" and "anti-cancer" were used as search terms in the GeneCards database (https://www. genecards.org/) [14], a searchable and integrative database that provides comprehensive and user-friendly information on all annotated and predicted human genes. The targets generated by the intersection between the tumor-related targets and predicted targets of $\mathrm{CBC}$ were identified as tumor-targets of CBC.

2.1.5. A Protein-Protein Interaction (PPI) Network Construction and Hub Targets Screening. The tumor-targets of CBC were submitted to STRING 11.0 database (https:// string-db.org/) [15], which stores information about protein interactions. Only proteins with the confidence score higher than 0.9 were selected. The protein information was imported into Cytoscape to construct the protein-protein interaction (PPI) network and merged with the herbcompounds-targets network. Finally, the hub targets and core compounds were screened by the values of degree and betweenness for constructing the core compound-target network. The hub targets were applied for enrichment and antitumor location analysis.

2.1.6. Network Construction of Nature and Flavor, Channel Tropism, and Tumor Location of CBC. Herbal drug properties in CBC were extracted from the Pharmacopoeia of the People's Republic of China according to the Four Properties (warm, cold, hot, and cool) and Five Flavors (pungent, sweet, sour, bitter, and salty) [16]. The nature and flavor (Chinese name, Xing wei), including Four Properties and Five Flavors, also called drug properties in herbal drug, which were clinically used according to clinical treatment experience of presented clinical symptom in patients in TCM theories. The channel tropism (Chinese name, Gui jing) is a theory of positioning and orientation of a certain organ, meridian, or specific clinical indication location to reveal the effect of TCM.

The nature and flavour and channel tropism of CBC were collected to construct a network via Cytoscape to analyze drug properties and clinical indications. Meanwhile, hub targets filtered by PPI network were uploaded to the Comparative Toxicogenomics Database (http://ctdbase.org/) to collect diseases' information. Diseases marked with "marker/mechanism" and defined as cancer were selected and imported into Cytoscape to explore the cluster site of $\mathrm{CBC}$ clinical indication.

2.1.7. Gene Ontology and Pathway Enrichment Analysis of $C B C$. To evaluate the role of targets by bioinformatic annotation, hub targets were imported into Enrichr database (http://amp.pharm.mssm.edu/Enrichr/) [17], which 
currently contains a large collection of diverse gene set libraries available for analysis. Hub targets were utilized by Enrichr high-throughput functional annotation bioinformatics to perform functional annotation, enrichment analysis, and gene ontology (GO) terms based on Kyoto Encyclopedia of Genes and Genomes (KEGG) pathways, with $P<0.05$. Terms and pathways were sorted based on combined scores, and target-pathway network was constructed via Cytoscape to display the tightly connection between targets and tumor-related pathways.

2.1.8. Integrative Mechanism Analysis of CBC in Tumor Treatment. To further explore potential mechanisms of CBC against tumor, networks of the core compound-target and target-pathway were merged to construct the herb-compound-target-pathway integrative network. The major tumor pathways were screened; among them, linked compounds and targets were identified as key compounds and targets subsequently. The integrative network was further verification of key compounds and targets, to integrate characteristics of multicompound, multitarget, and multipathway of CBC. Finally, the multivariate biological network was merged with the herb-compound-targetpathway, drug properties, and antitumor location network for comprehensive integration.

2.1.9. ADMET Absorption Level Analysis and Molecular Docking Simulation. Effective material basis of CBC was verified by ADMET absorption level and molecular docking. ADMET absorption level of compounds was obtained using the ETCM database. Levels of " $0,1,2$, and 3" meant "good, moderate, low, and very low," respectively. Key compounds with good ADMET absorption levels were selected for the integrative network analysis [18].

Molecular docking was performed to simulate structural stability between key compounds and targets using iGEMDOCK 2.1 software, a commonly used tool for molecular recognition, which evaluates and improves scoring functions [19]. The iGEMDOCK energy functions consist of electrostatic, steric, and hydrogen bonding potentials, which were used to recognize complexes. The key compounds in herb-compound-target-pathway network were downloaded using PubChem database (https://pubchem.ncbi.nlm.nih. gov/), energy minimized via Chem3D 19.0 software, and saved in MOL format. Protein targets were downloaded from PDB database (http://www.rcsb.org/). The standard docking settings were selected via iGEMDOCK: population size $=200$; generations $=70$; number of solutions $=2$. Results showed that lower energies correlated with more stable constructions. Finally, effective material basis of CBC was identified by ADMET absorption level analysis and molecular docking.

\subsection{Experimental Validation}

2.2.1. Cell Culture. Human SMMC-7721 cells (ATCC, USA) were chosen to validate our experiments. Cells were cultured in RPMI 1640 medium (Gibco, USA) supplemented with $10 \%$ FBS (Gibco, USA), $100 \mathrm{U} / \mathrm{ml}$ penicillin, and $100 \mathrm{mg} / \mathrm{ml}$ streptomycin (Solarbio, China) and maintained at $37^{\circ} \mathrm{C}$ in a humidified chamber under $5 \% \mathrm{CO}_{2}$.

2.2.2. Quantitative Real-Time PCR Analysis. The hub targets with higher degree, linked to hepatocellular carcinoma in the antitumor location network, were verified by qPCR. The SMMC-7721 cells $\left(4 \times 10^{6}\right.$ cells $)$ were seeded in $100 \mathrm{~mm}$ dishes and incubated for $24 \mathrm{~h}$. After, pretreatment with a range of $\mathrm{CBC}$ concentrations (Guizhou Yibai Pharmaceutical, China, Z52020238) (0, control; $0.01 \mathrm{mg} / \mathrm{ml}$, LCBC; $1 \mathrm{mg} / \mathrm{ml}, \mathrm{HCBC}$ ) in $12 \mathrm{~h}$ for RNA extraction was completed. Total RNA separation and extraction were performed according to the instructions of the TaKaRa MiniBEST Universal RNA Extraction Kit (TaKaRa, Clontech). Reverse transcription reactions were performed using $900 \mathrm{ng}$ of RNA with PrimerScript ${ }^{\mathrm{TM}}$ RT Master Mix (Perfect Real Time) for cDNA. Table 1 lists the primer sequences. Gene expression of PTGS2, TP53, ESR1, ABCB1, and TOP2A was investigated. The samples were exposed to predenaturation at $95^{\circ} \mathrm{C}$ for $30 \mathrm{~s}$, followed by 39 cycles of denaturation at $95^{\circ} \mathrm{C}$ for $5 \mathrm{~s}$, and at $60^{\circ} \mathrm{C}$ for $30 \mathrm{~s}$, and annealing at $95^{\circ} \mathrm{C}$ for $10 \mathrm{~s}$. The dissolution curve conditions were $65^{\circ} \mathrm{C}$ for $0.05 \mathrm{~s}$ and $95^{\circ} \mathrm{C}$ for $0.5 \mathrm{~s}$ using $10 \mu \mathrm{L}$ TB Green Premix EX Taq II, $0.8 \mu \mathrm{L}$ $10 \mu \mathrm{mol} / \mathrm{L}$ forward primer, $0.8 \mu \mathrm{L} 10 \mu \mathrm{mol} / \mathrm{L}$ reverse primer, and $2 \mu \mathrm{L}$ cDNA. Water was added to achieve a total volume of $6 \mu \mathrm{L}$. GAPDH was used as the internal control, and data were analyzed using the $2^{-\Delta \Delta \mathrm{Ct}}$ method. The experiment was repeated three times.

2.3. Statistical Analysis. Statistical analysis was performed using Prism 8.0.2 software. Data were expressed as means \pm SD and analyzed using Student's $t$-test. Differences between groups were considered to be statistically significant if $P<0.05$.

\section{Results}

\subsection{Network Pharmacology-Based Analysis}

3.1.1. Identification of Bioactive Compounds of CBC. After deleting duplicate data, a total of 128 compounds were identified as candidate compounds (Table 2), including 69 compounds with good QED and 69 effective literature-based antitumor compounds (Figures 1(a) and 1(b)) [20-36]. Cantharidin (CTD), a major component in BM, presents a strong antitumor activity [26], and major quality markers including specnuezhenide, liquiritin, loganin, and syringin of CBC were also identified [35].

3.1.2. Herb-Compound-Target Network Construction and Targets of $C B C$ against Tumors. In this study, 436 targets were screened against compounds from $\mathrm{CBC}$, which corresponded to an average of almost three targets per compound. The herb-compound-target network was constructed via Cytoscape software, where degree value changes with color and size variation (Figure 2). In order to 
Table 1: Primers used for quantitative real-time PCR (qPCR).

\begin{tabular}{lcc}
\hline Gene & Forward primer & Reverse primer \\
\hline$P T G S 2$ & CTGGCGCTCAGCCATACAG & CGCACTTATACTGGTCAAATCCC \\
TP53 & GAGGTTGGCTCTGACTGTACC & TCCGTCCCAGTAGATTACCAC \\
ESR 1 & GAAAGGTGGGATACGAAAAGACC 1 & GCTGTTCTTCTTAGAGCGTTTGA \\
TOP $2 A$ & GGGATGGTCAGTGTTGATGGA & GCTATCGTGGTGGCAAACAATA \\
GAPDH & ACCATTGCAGCCTGTAAATGA & GGGCGGAGCAAAATATGTTCC \\
\hline
\end{tabular}

Abbreviations. qPCR: quantitative real-time PCR; PTGS2: prostaglandin G/H synthase 2; TP53: cellular tumor antigen p53; ESR1: estrogen receptor; ABCB1: ATP-dependent translocase ABCB1; TOP2A: DNA topoisomerase 2-alpha.

TABle 2: Active compounds of compound banmao capsule (CBC).

\begin{tabular}{|c|c|c|}
\hline Herb & No. & Compound \\
\hline \multirow{8}{*}{ Mylabris (banmao) } & BM1 & Cantharidin \\
\hline & RS1 & Gomisin A \\
\hline & RS2 & Vitamin B1 \\
\hline & RS3 & Butylated hydroxytoluene \\
\hline & RS4 & A-Santalol \\
\hline & RS5 & Deoxygomisin A \\
\hline & RS6 & 2,5-Dimethyl-7-hydroxy chromone \\
\hline & RS7 & Ginsenoside rc \\
\hline \multirow{13}{*}{ Ginseng Radix Et Rhizoma (ren shen) } & RS8 & Ginsenoside rd \\
\hline & RS9 & Ginsenoside Re \\
\hline & RS10 & Ginsenoside Rg3 \\
\hline & RS11 & Ginsenoside Rh4 \\
\hline & RS12 & Ginsenoside Rh2 \\
\hline & RS13 & Kaempferol \\
\hline & RS14 & Ginsenoside Rb1 \\
\hline & RS15 & Ginsenoside Rg1 \\
\hline & CWJ1 & Sesamin \\
\hline & CWJ2 & Neociwujiaphenol \\
\hline & CWJ3 & Syringic acid \\
\hline & CWJ4 & Syringaresinol \\
\hline & CWJ5 & 3-O-trans-ferulylquinic acid \\
\hline \multirow{8}{*}{$\begin{array}{l}\text { Acanthopanacis Senticosi Radix Et Rhizoma } \\
\text { Seu Caulis (ci wu jia) }\end{array}$} & CWJ6 & Hederasaponin B \\
\hline & CWJ7 & Eleutheroside K \\
\hline & CWJ8 & Ciwujianoside $\mathrm{C} 1$ \\
\hline & CWJ9 & Ciwujianoside D1 \\
\hline & CWJ10 & Ciwujianoside B \\
\hline & CWJ11 & Ciwujianoside D2 \\
\hline & CWJ12 & Syringin \\
\hline & CWJ13 & Isofraxidin \\
\hline
\end{tabular}


TABle 2: Continued.

\begin{tabular}{|c|c|c|}
\hline Herb & No. & Compound \\
\hline \multirow{24}{*}{ Curcumae Rhizoma (e zhu) } & $\mathrm{EZ1}$ & 7-Hydroxy-5-methoxyflavanone \\
\hline & EZ2 & Pinocembrin \\
\hline & EZ3 & Curcarabranol A \\
\hline & $\mathrm{EZ4}$ & $\begin{array}{c}\text { (1S,3 R,6R,7 R)-1-Methyl-7-(2-(2-methyl-1,3-dioxolan-2-Yl) ethyl)-4-(propan-2- } \\
\text { ylidene) bicyclo [4.1.0] Heptan-3-ol }\end{array}$ \\
\hline & EZ5 & $\begin{array}{c}\text { (4Ar,5R,5 As,6Ar)-6a-Hydroxy-3,5a-dimethyl-5-(3-oxobutyl)-4,4A,5,5A,6,6A- } \\
\text { hexahydro-2h-cyclopropa (F) [1] benzofuran-2-one }\end{array}$ \\
\hline & EZ6 & $\begin{array}{c}\text { (5S,8 R,9S,10S,13S,14S)-3-Ethyl-3-hydroxy-10,13-dimethyl-tetradecahydro-2h- } \\
\text { cyclopenta [A] phenanthren-17 (14H)-one }\end{array}$ \\
\hline & EZ7 & $\begin{array}{c}\text { (8S,8 As)-8-Hydroxy-3,5,8a-trimethyl-7,8,8A,9-tetrahydronaphtho }[2,3 \mathrm{~B}] \text { furan-4 } \\
(6 \mathrm{H}) \text {-one }\end{array}$ \\
\hline & EZ8 & Dihydrocurcumenone \\
\hline & EZ9 & $\begin{array}{c}\text { 4-((1S,6 R,7R)-4-(2-Hydroxypropan-2-yl)-1-methylbicyclo [4.1.0] hept-3-en-7-Yl) } \\
\text { butan-2-one }\end{array}$ \\
\hline & EZ10 & $\begin{array}{c}\text { (4Ar,5R,5 As,6 As)-3,5a-Dimethyl-5-(3-oxobutyl)-4,4A,5,5A,6,6A-hexahydro-2h- } \\
\text { cyclopropa (F) [1] benzofuran-2-one }\end{array}$ \\
\hline & EZ11 & Epicurzerenone \\
\hline & EZ12 & Curzerenone \\
\hline & EZ13 & Zedoarol \\
\hline & EZ14 & $\begin{array}{c}\text { (3S,3 As,5S,8 As)-3a-Hydroxy-3,3', } 3^{\prime}, 8 \text {-tetramethyl-1,2,3,3A,4,8A-hexahydro- } 6 \mathrm{~h} \text { - } \\
\text { spiro [azulene-5, } 2^{\prime} \text {-oxiran]-6-one }\end{array}$ \\
\hline & EZ15 & Curcumenone \\
\hline & EZ16 & (S)-2-Methyl-6-(4-methylcyclohex-3-en-1-Yl) hepta-2,6-dien-1-ol \\
\hline & $\mathrm{EZ17}$ & Ar-Turmerone \\
\hline & EZ18 & Isovelleral \\
\hline & EZ19 & Azulen-5-ylmethanol \\
\hline & EZ20 & $\beta$-Elemene \\
\hline & $\mathrm{EZ21}$ & Curcumol \\
\hline & EZ22 & Curdione \\
\hline & NZZ1 & $\beta$-Sitosterol \\
\hline & NZZ2 & Quercetin \\
\hline \multirow{8}{*}{ Ligustri Lucidi Fructus (nv zhen zi) } & NZZ3 & Ursolic acid \\
\hline & NZZ4 & Oleanolic acid \\
\hline & NZZ5 & Specnuezhenide \\
\hline & NZZ6 & Ligustroflavone \\
\hline & XD1 & Ursodeoxycholic acid \\
\hline & $\mathrm{XD} 2$ & Tauroursodeoxycholic acid \\
\hline & XD3 & Deoxycholic acid \\
\hline & XD4 & Cholic acid \\
\hline \multirow[t]{5}{*}{ Fel Ursi (xiong dan) } & XD5 & Taurochenodeoxycholic acid \\
\hline & XD6 & Chenodeoxycholic acid \\
\hline & $\mathrm{XD} 7$ & 4,7-Dihydroxyisoflavone \\
\hline & $\mathrm{XD} 8$ & 4,5,7-Trihydroxyiso-flavone \\
\hline & XD9 & 4,7-Dihydroxy-6-methoxyflavone \\
\hline \multirow{7}{*}{ Glycyrrhizae Radix Et Rhizoma (gan cao) } & GC32 & Glabridin \\
\hline & GC33 & Licoricidin \\
\hline & HQ1 & Kumatakenin \\
\hline & HQ2 & Medicarpin \\
\hline & HQ3 & Isorhamnetin \\
\hline & HQ4 & Calycosin \\
\hline & HQ5 & Formononetin \\
\hline \multirow[t]{6}{*}{ Astragali Radix (huang qi) } & HQ6 & Quercetin \\
\hline & HQ7 & Kaempferol \\
\hline & HQ8 & 7-O-Methylisomucronulatol \\
\hline & HQ9 & Astragaloside iv \\
\hline & HQ10 & Astragaloside II \\
\hline & HQ11 & Astramembrannin ii \\
\hline
\end{tabular}


TABLE 2: Continued.

\begin{tabular}{|c|c|c|}
\hline Herb & No. & Compound \\
\hline \multirow{7}{*}{ Sparganii Rhizoma (san leng) } & SL1 & Kaempferol \\
\hline & SL2 & Formononetin \\
\hline & SL3 & Hederagenin \\
\hline & SL4 & $\beta$-Sitosterol \\
\hline & SL5 & Stigmasterol \\
\hline & SL6 & Trans-gondoic acid \\
\hline & BZL1 & 7-Hydroxy-5,8-dimethoxyflavone \\
\hline \multirow{8}{*}{ Scutellariae Barbatae Herba (ban zhi lian) } & BZL2 & Wogonin \\
\hline & BZL3 & Rivularin \\
\hline & BZL4 & 4'-Hydroxywogonin,5,7-dihydroxy-8-methoxylflavone \\
\hline & BZL5 & p-Hydroxybenzyl acetone \\
\hline & BZL6 & Baicalein \\
\hline & BZL7 & Scutebarbatines A \\
\hline & BZL8 & Scutebarbatines B \\
\hline & BZL9 & Scutellarin \\
\hline \multirow{9}{*}{ Corni Fructus (shan zhu yu) } & SZY1 & Isoasarone \\
\hline & SZY2 & Elemicin \\
\hline & SZY3 & 1-Allyl-2,4,5-trimethoxy-benzene \\
\hline & SZY4 & Ethylvanillin \\
\hline & SZY5 & Asaricin \\
\hline & SZY6 & Retinol \\
\hline & SZY7 & Iridoid \\
\hline & SZY8 & Loganin \\
\hline & SZY9 & Morroniside \\
\hline \multirow{33}{*}{ Glycyrrhizae Radix Et Rhizoma (gan cao) } & GC1 & Formononetin \\
\hline & GC2 & $3^{\prime}, 7$-Dihydroxy-4',6-dimethoxyisoflavone \\
\hline & GC3 & Kumatakenin \\
\hline & GC4 & Gancaonin $\mathrm{X}$ \\
\hline & GC5 & $4^{\prime}$-O-Methylglabridin \\
\hline & GC6 & Gancaonin Y \\
\hline & GC7 & $3^{\prime}$-Methoxyglabridin \\
\hline & GC8 & Glycyrrhisoflavanone \\
\hline & GC9 & Glyzaglabrin \\
\hline & GC10 & Phaseollinisoflavan \\
\hline & GC11 & (S)-5,7-Dihydroxy-2-phenylchroman-4-one, pinocembrin \\
\hline & GC12 & Liquiritigenin \\
\hline & GC13 & Licoagroisoflavone \\
\hline & GC14 & Licoagropin \\
\hline & GC15 & Gancaonin A \\
\hline & GC16 & Maackiain \\
\hline & GC17 & $3,3^{\prime}$-Dimethylquercetin \\
\hline & GC18 & Erythrinin C \\
\hline & GC19 & Gancaonin $\mathrm{Z}$ \\
\hline & GC20 & Licoricone \\
\hline & GC21 & Hispaglabridin B \\
\hline & GC22 & Lupiwighteone \\
\hline & GC23 & Semilicoisoflavone B \\
\hline & GC24 & Licoisoflavone B \\
\hline & GC25 & Xambioona \\
\hline & GC26 & Gancaonin B \\
\hline & GC27 & Glicoricone \\
\hline & GC28 & Glycyrrhizic acid \\
\hline & GC29 & Glycyrrhetinic acid \\
\hline & GC30 & Isoliquiritigenin \\
\hline & GC31 & Licochalcone A \\
\hline & GC34 & Isoangustone $\mathrm{A}$ \\
\hline & GC35 & Liquiritin \\
\hline
\end{tabular}

Abbreviations. QED: quantitative estimate of drug-likeness; BM: Mylabris; RS: Ginseng Radix Et Rhizoma; HQ: Astragali Radix; CWJ: Acanthopanacis Senticosi Radix Et Rhizoma Seu Caulis; SL: Sparganii Rhizoma; EZ: Curcumae Rhizoma; BZL: Scutellariae Barbatae Herba; SZY: Corni Fructus; NZZ: Ligustri Lucidi Fructus; XD: Fel Ursi; GC: Glycyrrhizae Radix Et Rhizoma. 


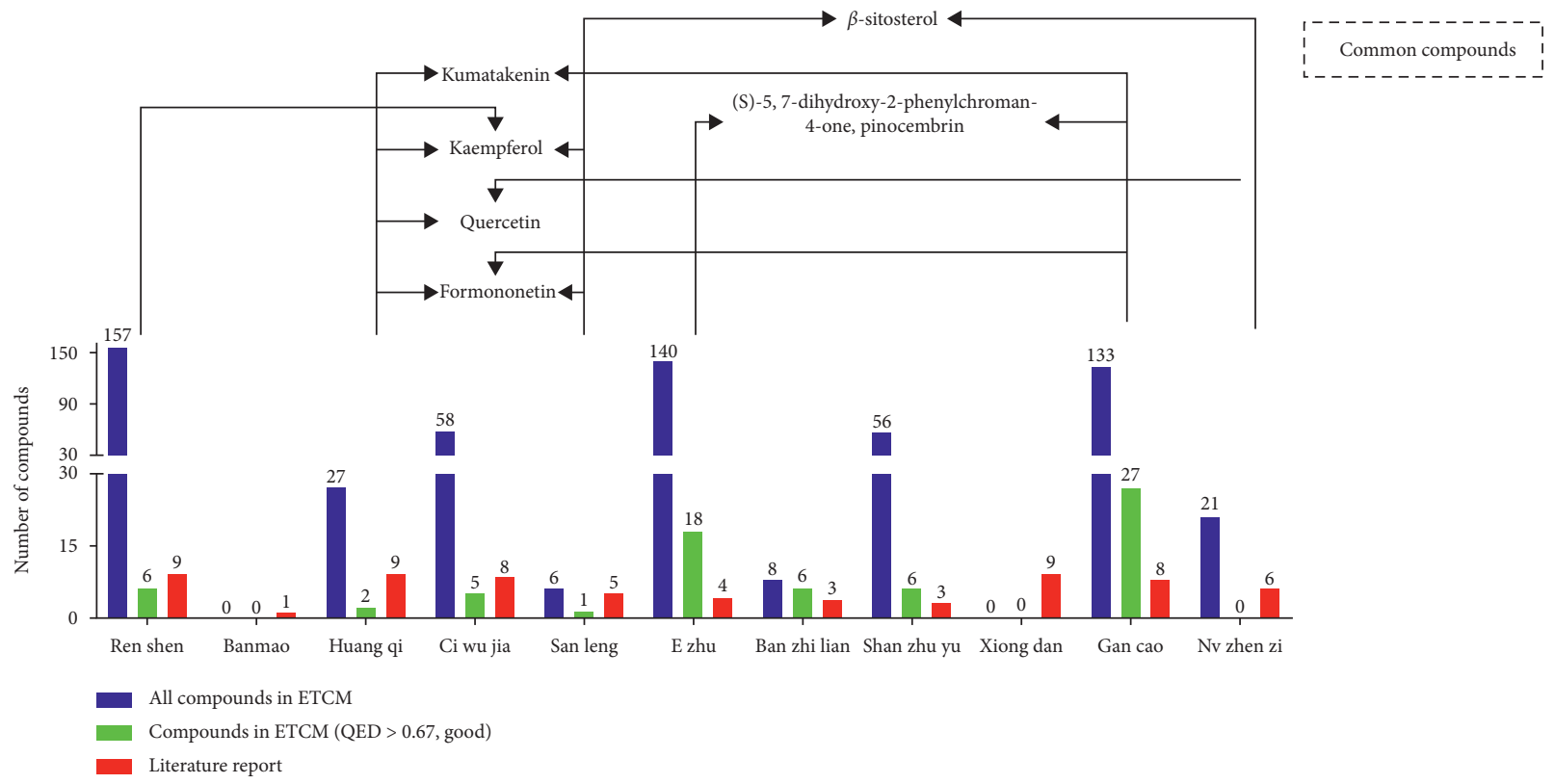

(a)

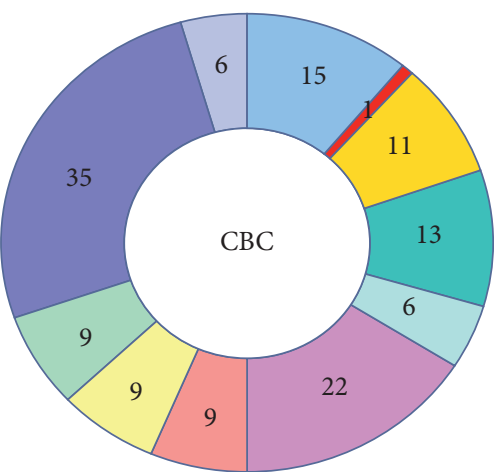

Total compounds $=128$

$\begin{array}{ll}\square \text { Ren shen } & \square \text { Ban zhi lian } \\ \text { Ban mao } & \square \text { Shan zhu yu } \\ \square \text { Huang qi } & \square \text { Xiong dan } \\ \square \text { Ci wu jia } & \square \text { Gan cao } \\ \square \text { San leng } & \square \text { Nv zhen zi } \\ \square \text { E zhu } & \end{array}$

(b) 


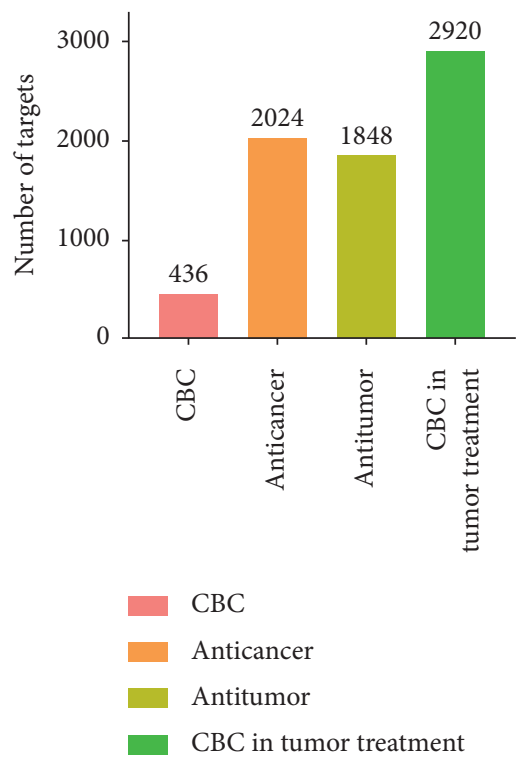

(c)

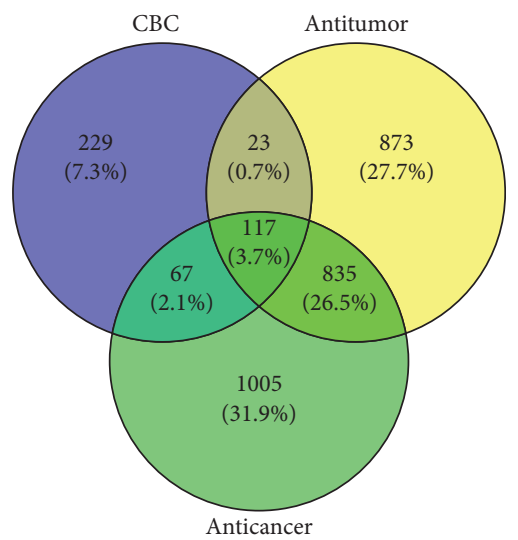

(d)

Figure 1: Diagram of compounds and targets in compound banmao capsule (CBC) against tumors. (a) Number of compounds of 11 herbs from CBC. (b) Proportion of compounds of each herb from CBC. (c) Number of targets in CBC and predicted tumor-targets. (d) Venn diagram of targets in $\mathrm{CBC}$ against tumors.

identify correlation between each herb in CBC, a heat map was applied to reveal common number of targets. EZ correlated with 242 targets of $\mathrm{CBC}$, while banmao was connected to 49 targets (Figure 3). Alternatively, by searching "anti-tumor" and "anti-cancer" in the GeneCards database, 1848 and 2024 targets were obtained, respectively. The 2920 common targets were selected (Figure 1(c)). Subsequently, the intersection of 207 CBC tumor targets was filtered out between disease targets and CBC targets (Figure 1(d)).

3.1.3. PPI and Core Compound-Target Network Construction. Tumor-targets of CBC were brought to the String database to perform a PPI network with the highest confidence (0.9). A total of 158 nodes and 619 edges were obtained in the PPI network (Figure 4). The PPI network was merged with the herb-compounds-targets network to build the core compound-target network based on topological parameters with average degree value $>18.43$ and average betweenness >
0.007. Then, 39 putative hub targets and 29 core compounds were identified (Figure 5), suggesting that the hub targets and compounds had multiple beneficial biological functions for treating tumors at the molecular level.

3.1.4. Drug Properties and Organism Location of Antitumor Analysis. Based on Pharmacopoeia of the People's Republic of China, the 11 herbs of CBC were categorized according to the Four Properties and Five Flavors and imported into Cytoscape (Figure 6). Based on topological analysis, the pungent $($ degree $=5)$, bitter $\quad($ degree $=5)$, and sweet $($ degree $=4)$ presented a higher degree value in nature and flavor. In addition, the liver-meridian (degree $=7$ ), kidneymeridian $($ degree $=6)$, and spleen-meridian $($ degree $=6)$ had a higher degree value in channel tropism.

A total of 96 diseases containing 13 categories of tumors were collected by enrichment analysis of 39 hub targets. Detail mapping relations were visualized via Cytoscape 


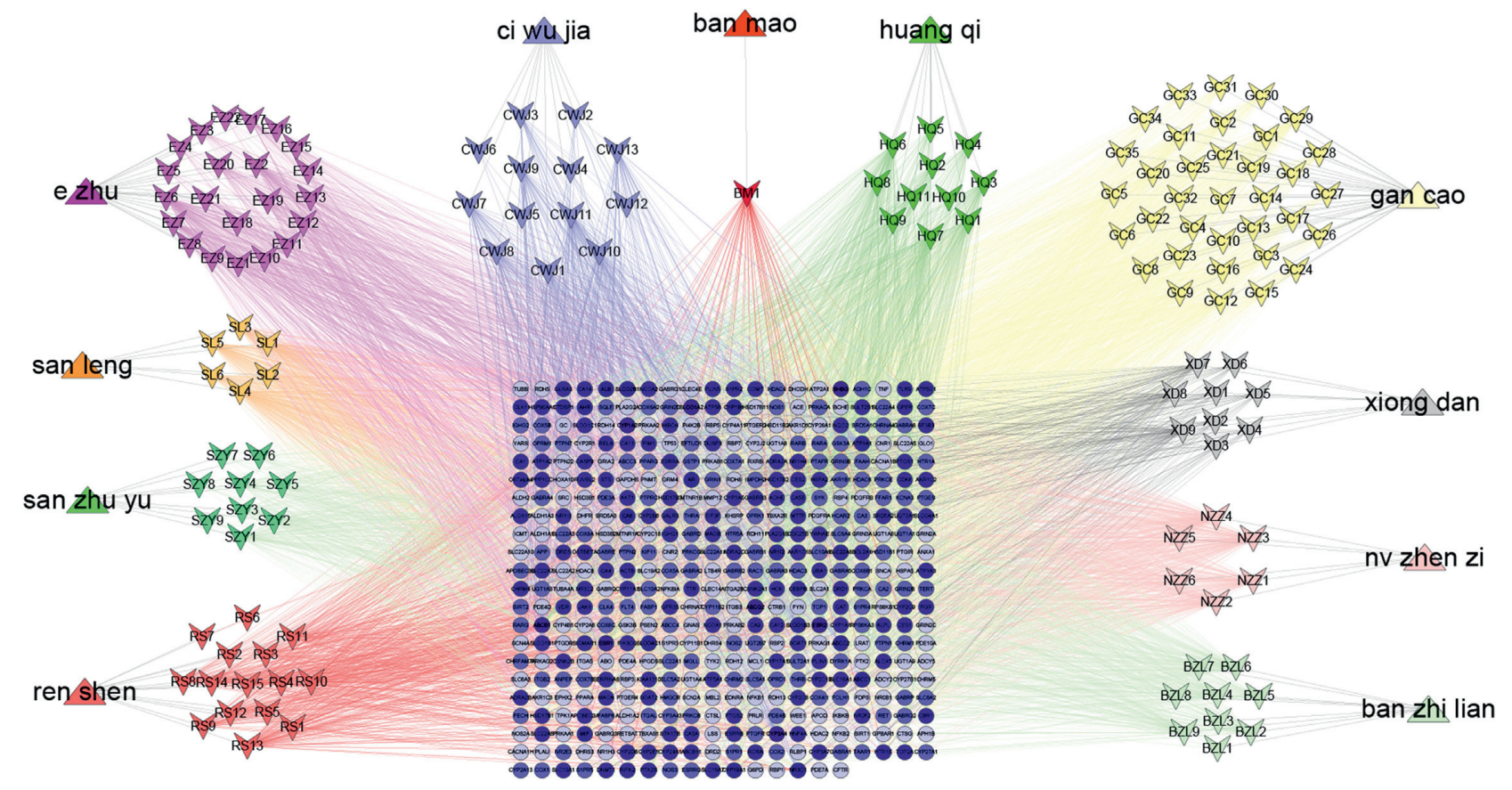

FIgURe 2: Herb-compound-targets network in compound banmao capsule (CBC). Triangle, quadrilateral, and round shapes stand for herb, compound, and target, respectively. Degree value changes with color and size variation.

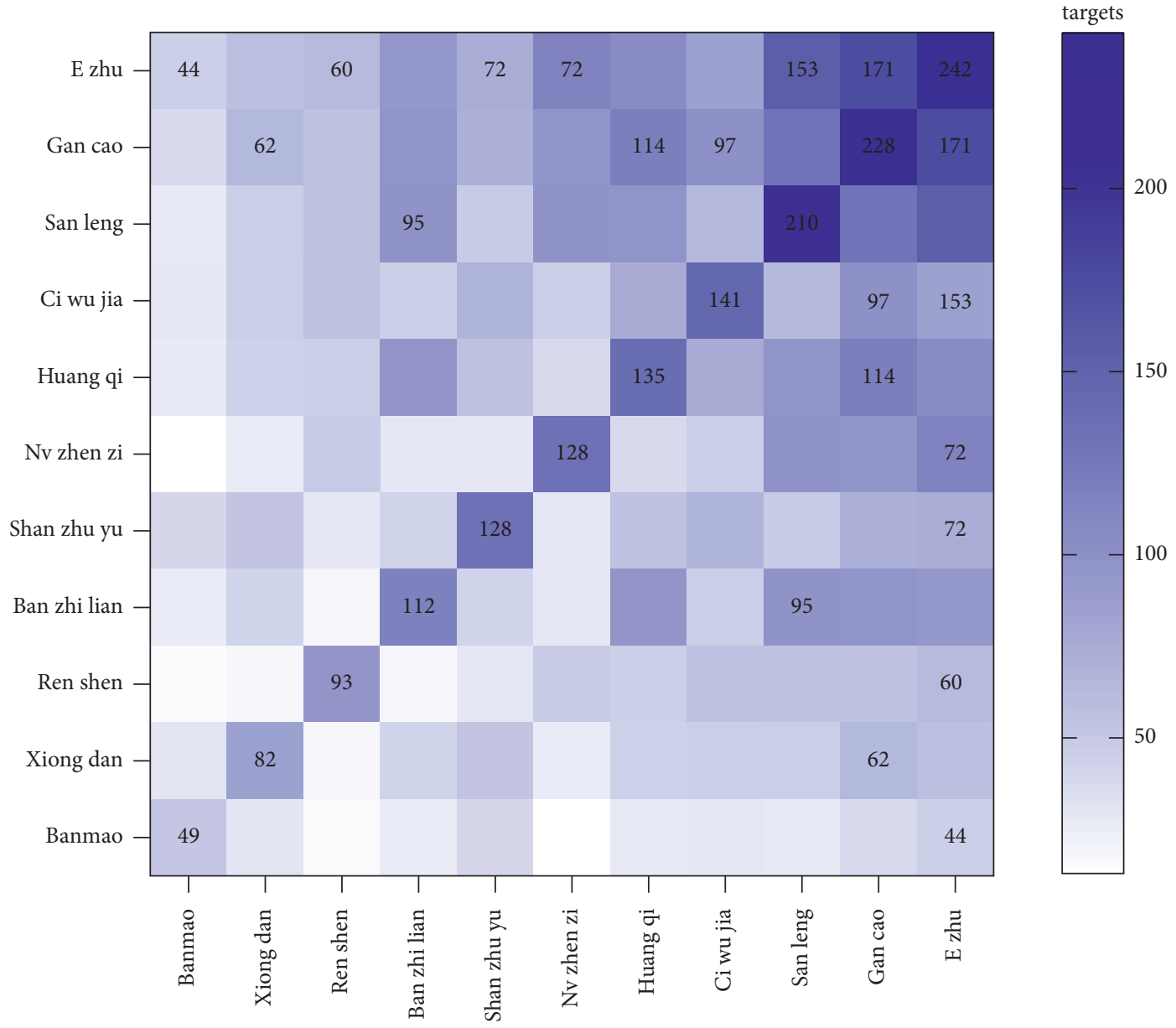

Figure 3: Intersection targets in each herb from compound banmao capsule (CBC). $X$ axis and $Y$ axis stand for herbs from CBC. The most intersection targets between two herbs from $\mathrm{CBC}$ were shown with number in the figure, and number of intersection target changes with color variation. 


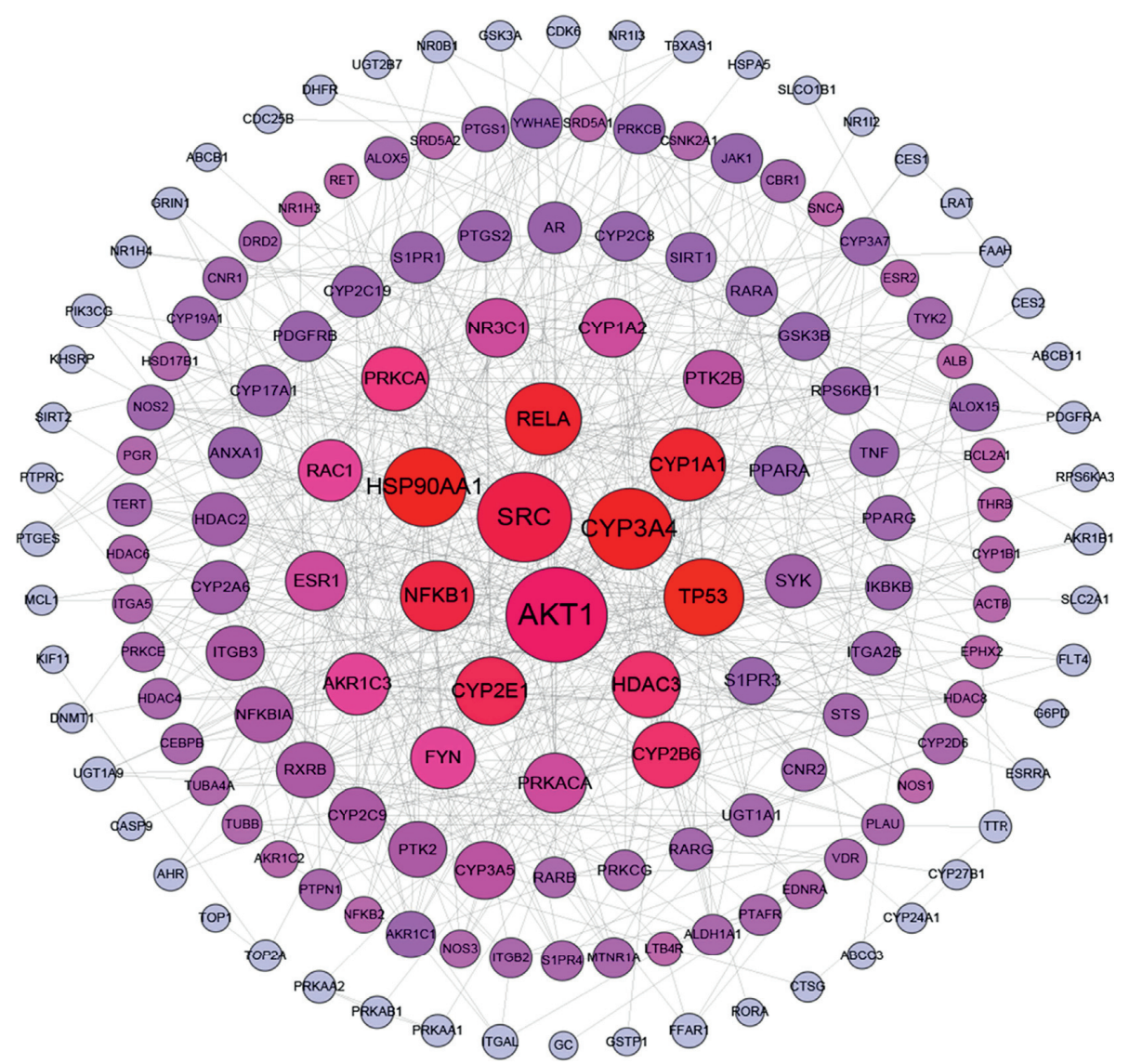

(a)

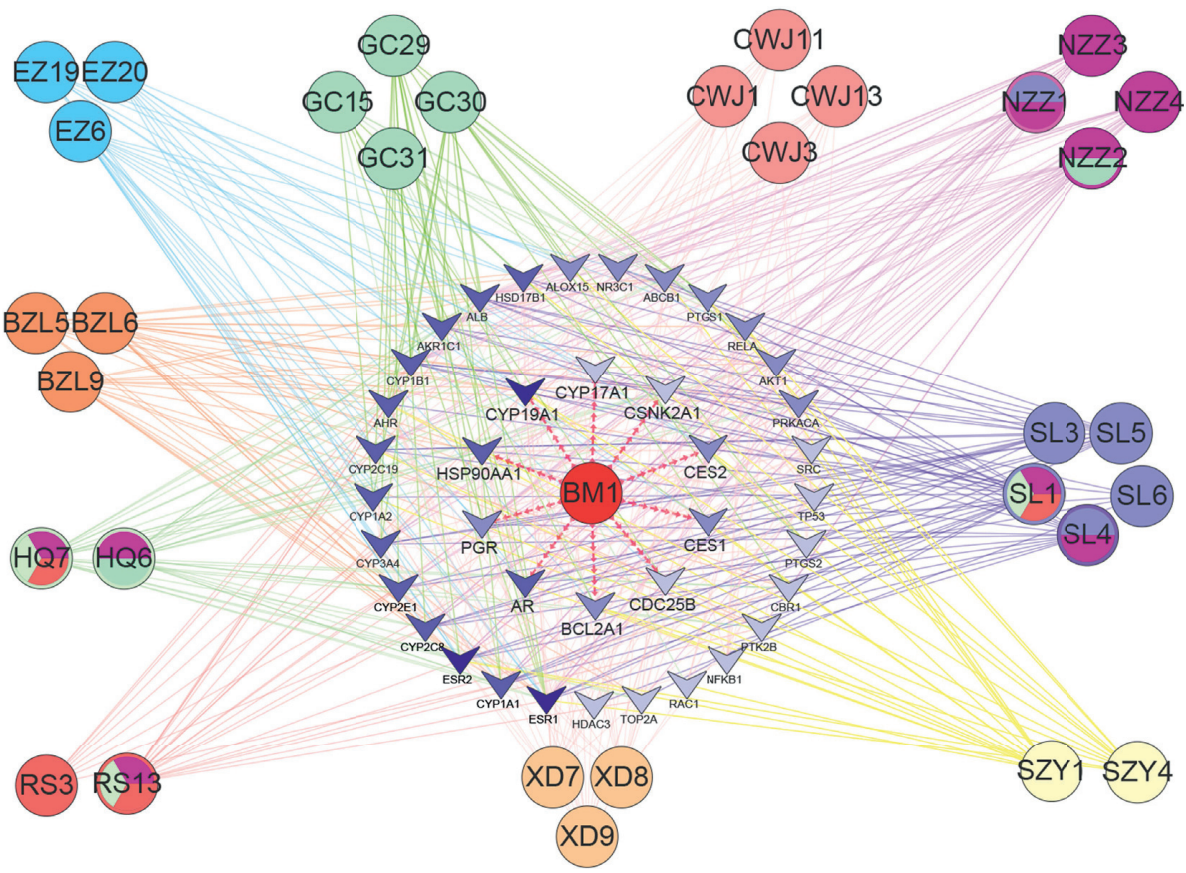

(b)

Figure 4: (a) Protein-protein interaction (PPI) network in compound banmao capsule (CBC) targets against tumors. Colors from blue to red and size change indicate that the degree value is increasing. (b) Core-compound-target network in CBC against tumors. Quadrilateral and round shapes stand for target and compound, respectively. Nodes and edges with the same color represent the same herb and related targets. Color from shallow to deep; the target degree value was increasing. 


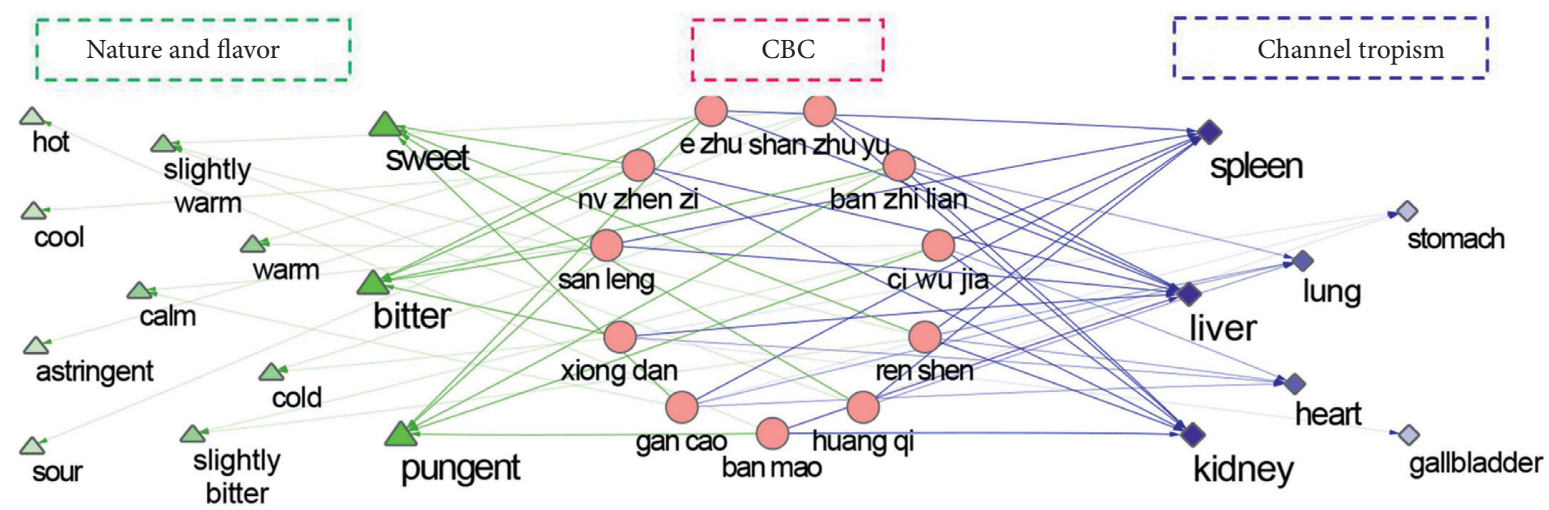

(a)

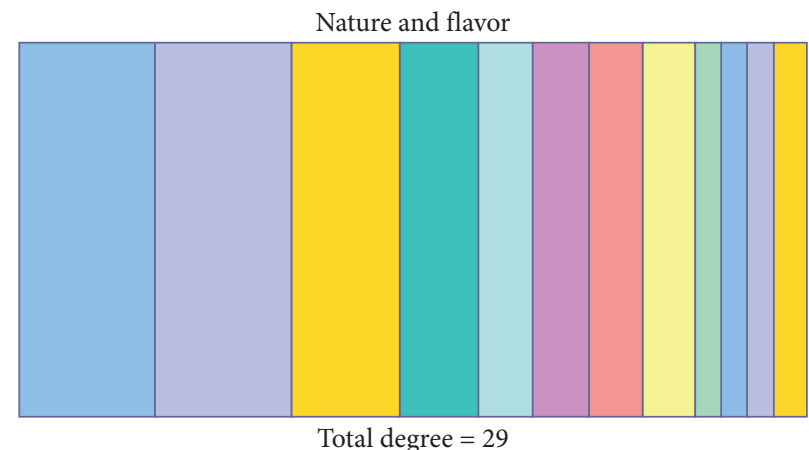

Total degree $=29$

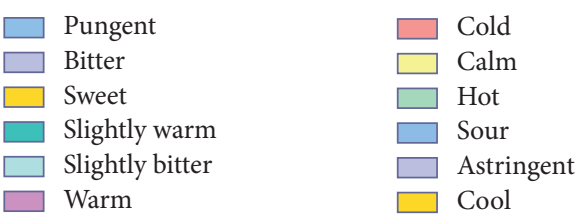

(b)

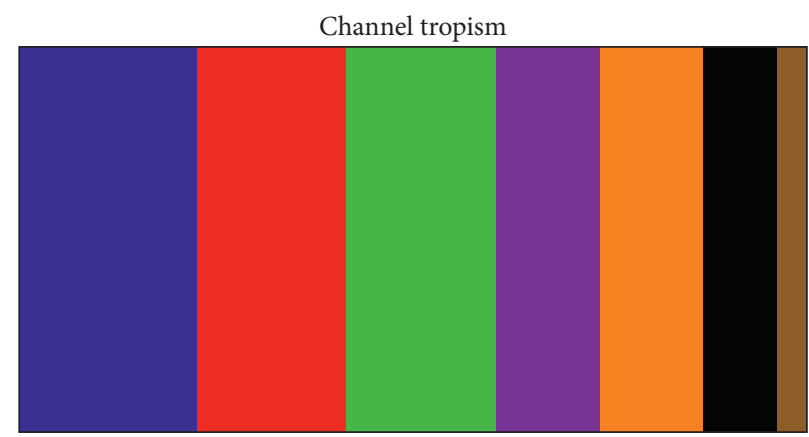

Total degree $=31$

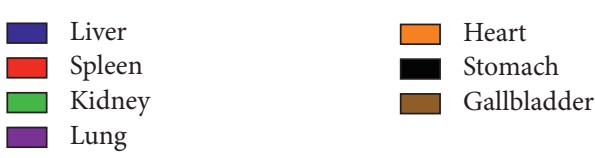

(c)

FIGURE 5: Nature and flavor and channel tropism in compound banmao capsule (CBC). (a) Detail mapping information of each herb from CBC. Green, red, and blue stand for nature and flavor, herbs from CBC, and channel tropism, respectively. (b) Detail degree value proportion in nature and flavor in herbs form CBC. (c) Detail degree value proportion in channel tropism in herbs form CBC.

(Figure 7). Among them, the targets TP53 (degree $=58$ ), PTGS2 (degree $=40)$, and ESR1 (degree=20) showed a higher degree value in tumor location. Fourteen types of diseases were classified as cancer of digestive and urogenital systems. Compared with other diseases, breast neoplasms $($ degree $=21)$, prostatic neoplasms $($ degree $=19)$, and 


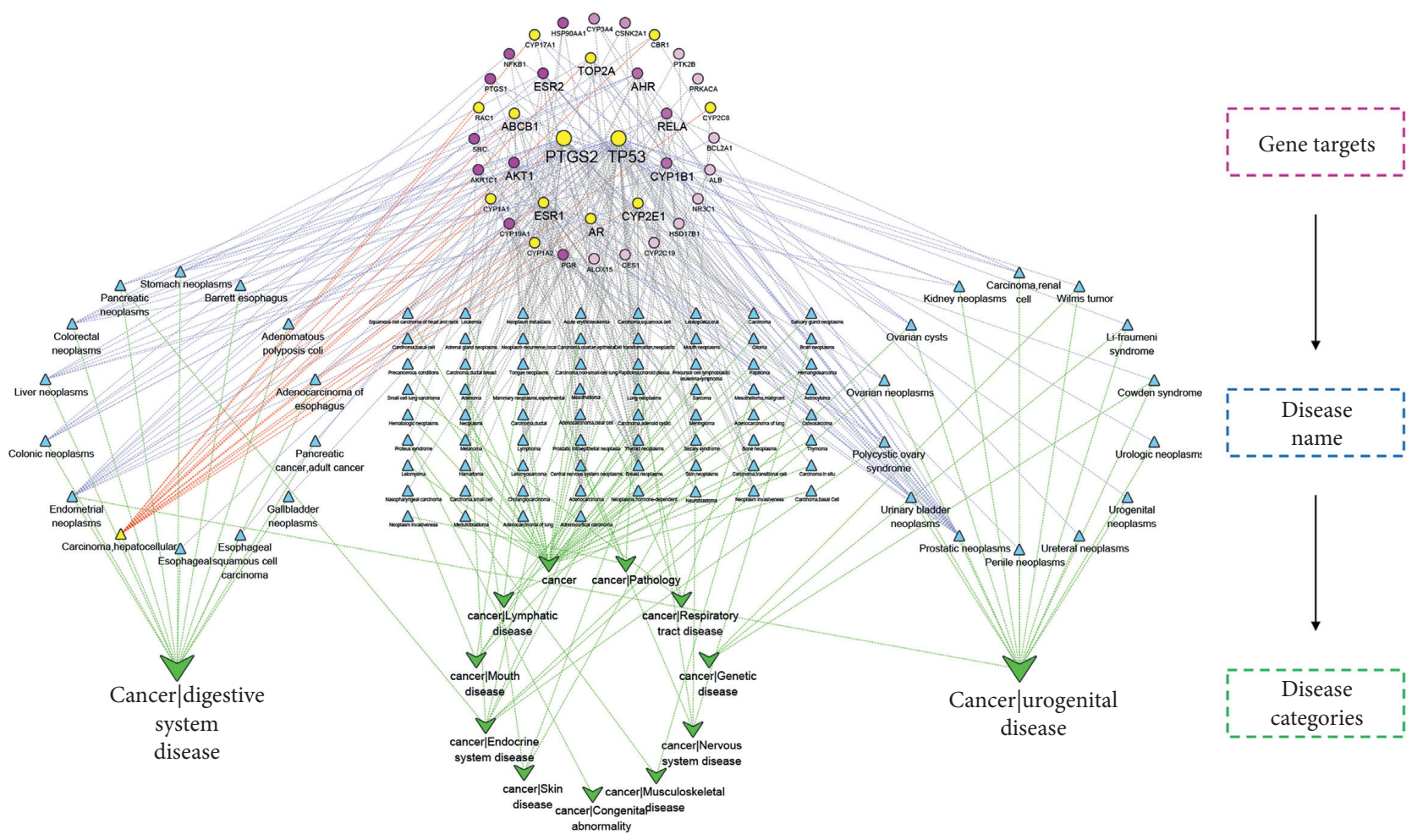

FIGURE 6: Antitumor location network in compound banmao capsule (CBC) against tumors. Round, triangle, and quadrilateral shapes stand for target, disease name, and disease categories. Yellow nodes stand for targets in hepatocellular carcinoma.

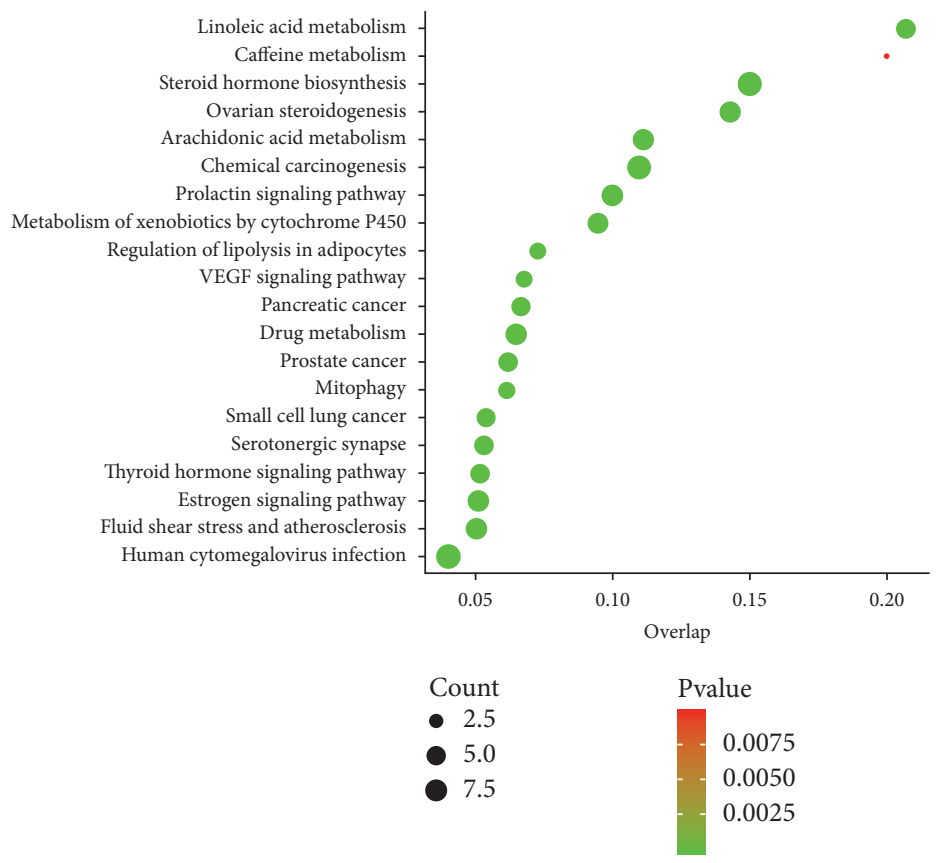

(a)

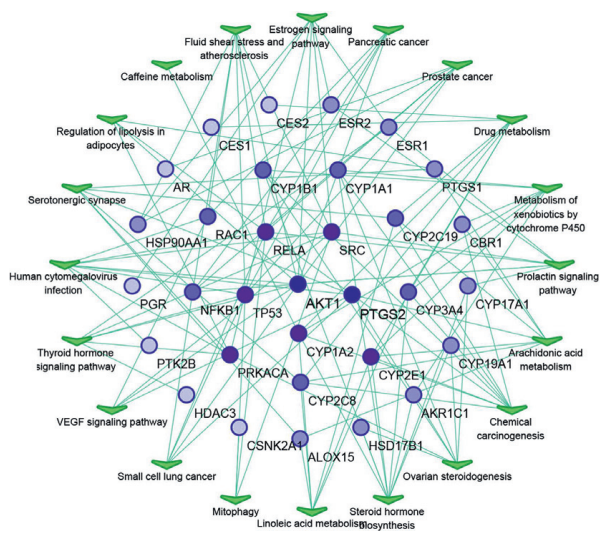

(b)

Figure 7: Continued. 


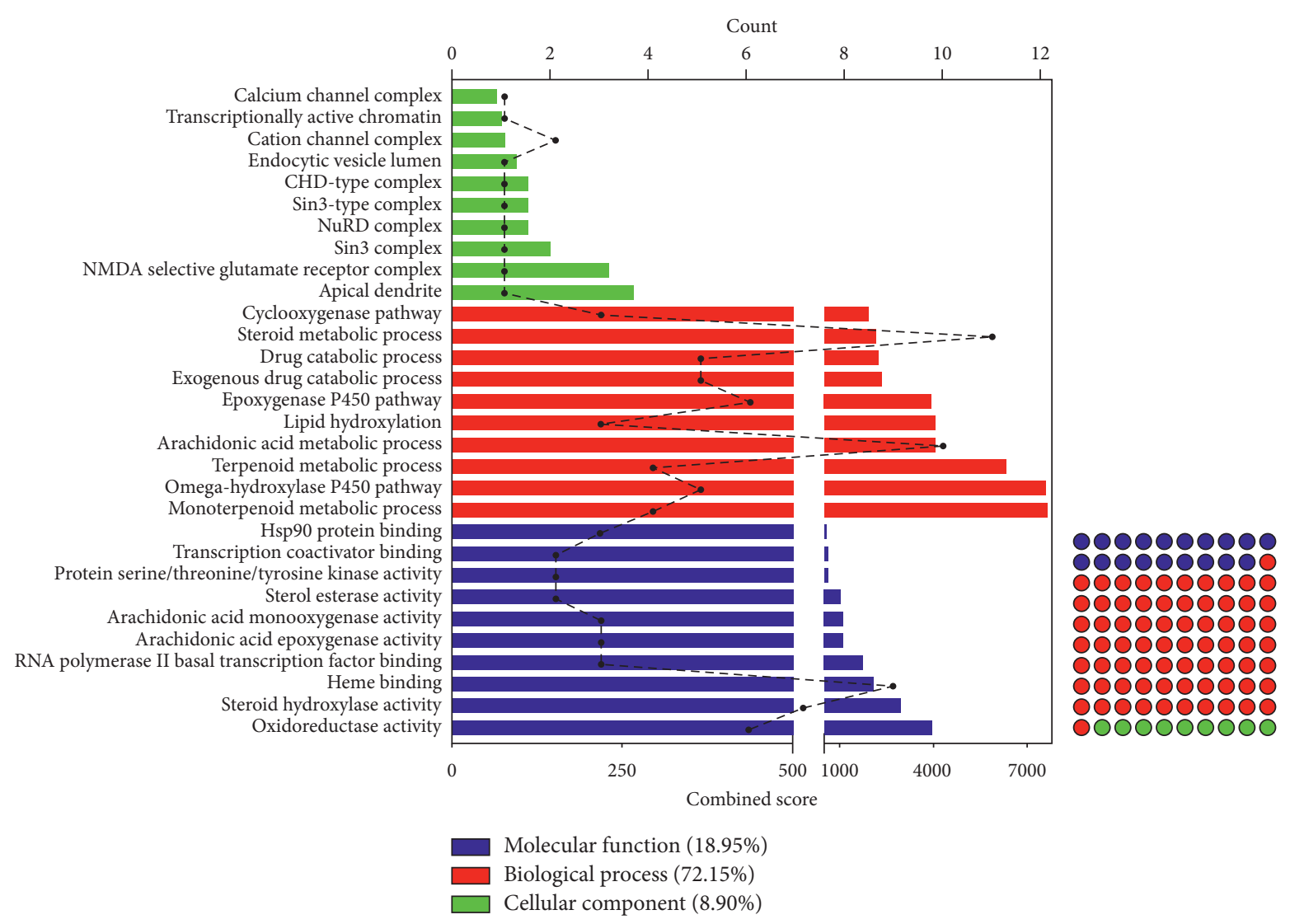

(c)

FIGURE 7: Enrichment analysis of targets in compound banmao capsule (CBC) against tumors. (a) Bubble plot of KEGG pathway analysis in CBC against tumors. $X$ axis and $Y$ axis stand for overlap and pathways respectively. (b) Target-pathway network in CBC. Round shape stands for count target; quadrilateral stands for pathway. Colors varying from shallow to deep indicate that the target degree value is increasing. (c) GO functional enrichment analysis in CBC against tumors. Bottom $X$ axis, top $X$ axis, and $Y$ axis stand for combined score, count gene, and GO term. Green, red, and blue stand for cellular component (CC), biological processes (BP), and molecular function (MF), respectively.

hepatocellular carcinoma (degree $=14$ ) presented higher degree values. In general, the antitumor activity of CBC may have a superior efficacy in liver, kidney, and breast tumors.

3.1.5. GO and Pathway Enrichment Analysis. Thirty-nine hub targets were imported into Enrichr database enrichment analysis. The top $20 \mathrm{KEGG}$ pathways $(P<0.05)$ were visualized by bubble plot (Figure $7(\mathrm{a})$ ), and target-pathway network was built using Cytoscape, obtaining 32 nodes of targets (Figure 7(b)). The top $10 \mathrm{GO}$ enrichment analyses were performed individually $(P<0.05)$, including cellular component (CC), molecular function (MF), and biological processes (BP), which were ranked by a combined score (Figure $7(\mathrm{c})$ ).

In this study, 132 pathways were enriched $(P<0.05)$, suggesting that the putative targets were highly connected with the regulation of prostate cancer, pancreatic cancer, estrogen signaling pathway, vascular endothelial growth factor (VEGF) signaling pathway, small cell lung cancer, and mitophagy. Alternatively, 290 GO terms were obtained $(P<0.05)$, including estrogen metabolic process, prostaglandin metabolic process, cellular response to angiotensin, estrogen biosynthetic process, and prostaglandin biosynthetic process, suggesting that $\mathrm{CBC}$ may influence cancer-related pathways, mitophagy, angiogenesis, and metabolism of estrogen and prostaglandin for tumor treatment.

\subsubsection{Multivariate Biological Network Build and Verification} Analysis. The herb-compound-target-pathway network was constructed using the target-pathway network and core compound-target network (Figure 8). The integrative network comprised 11 herbs, 26 key compounds, 13 hub targets, and five pathways, suggesting that the characteristics of multicompound-target-pathway of $\mathrm{CBC}$, and each herbal drug was indispensable in tumor treatment. Among them, VEGF signaling pathway, estrogen signaling pathway, small cell lung cancer, pancreatic cancer, and prostate cancer were selected as mainly tumor-related pathways. Further integration of the multivariate biological network was merged based on VEGF and estrogen signaling pathways, involving nine targets, which are linked with 10 digestive system 


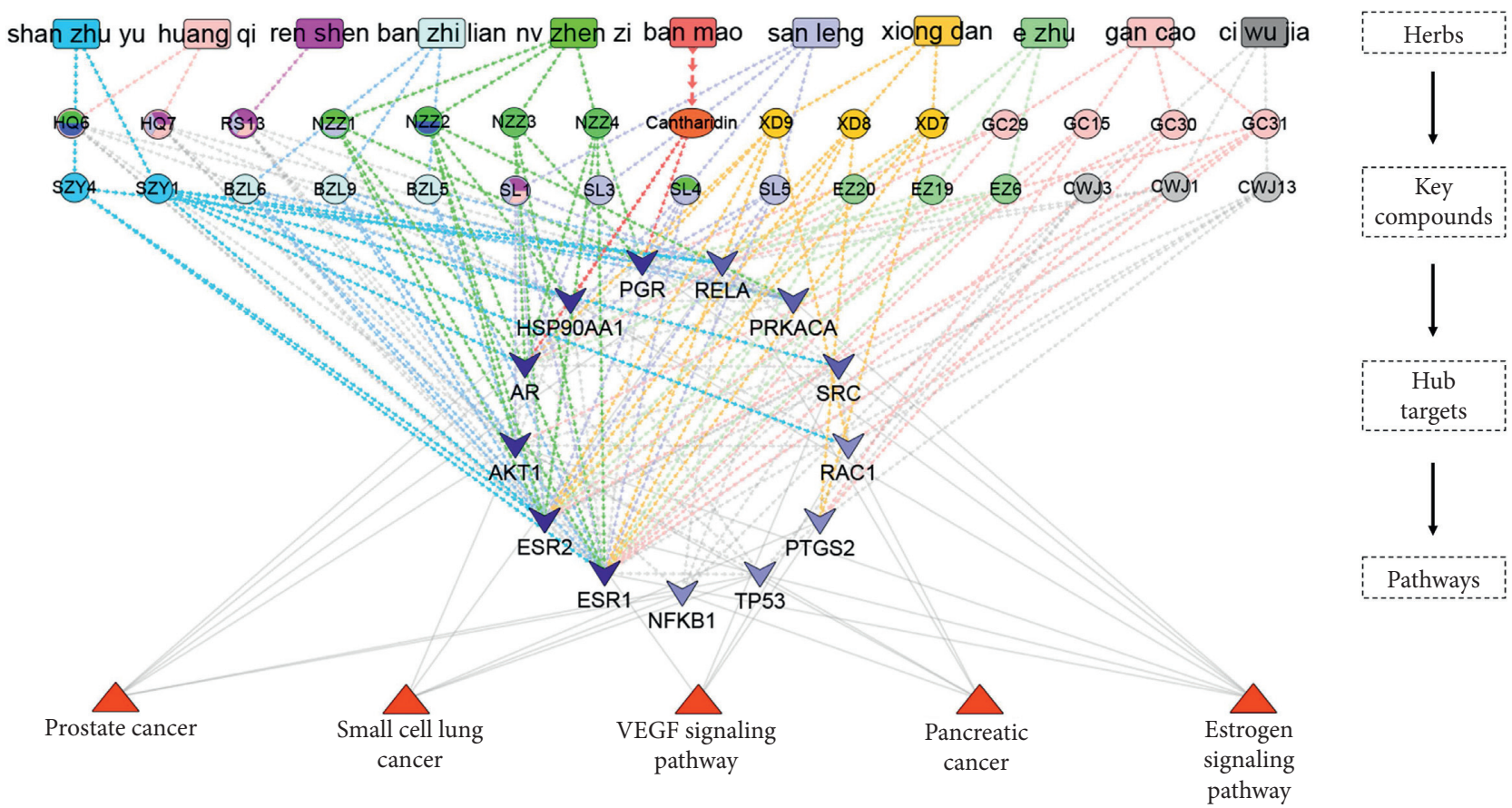

Figure 8: Herb-compound-target-pathway network of compound banmao capsule (CBC) against tumors. From top to bottom stand for herbs, key compounds, hub targets, and pathways, respectively. Nodes and edges of compounds come from the same herb and are defined with the same color. Colors varying from shallow to deep indicate that the target degree value is increasing in hub targets.

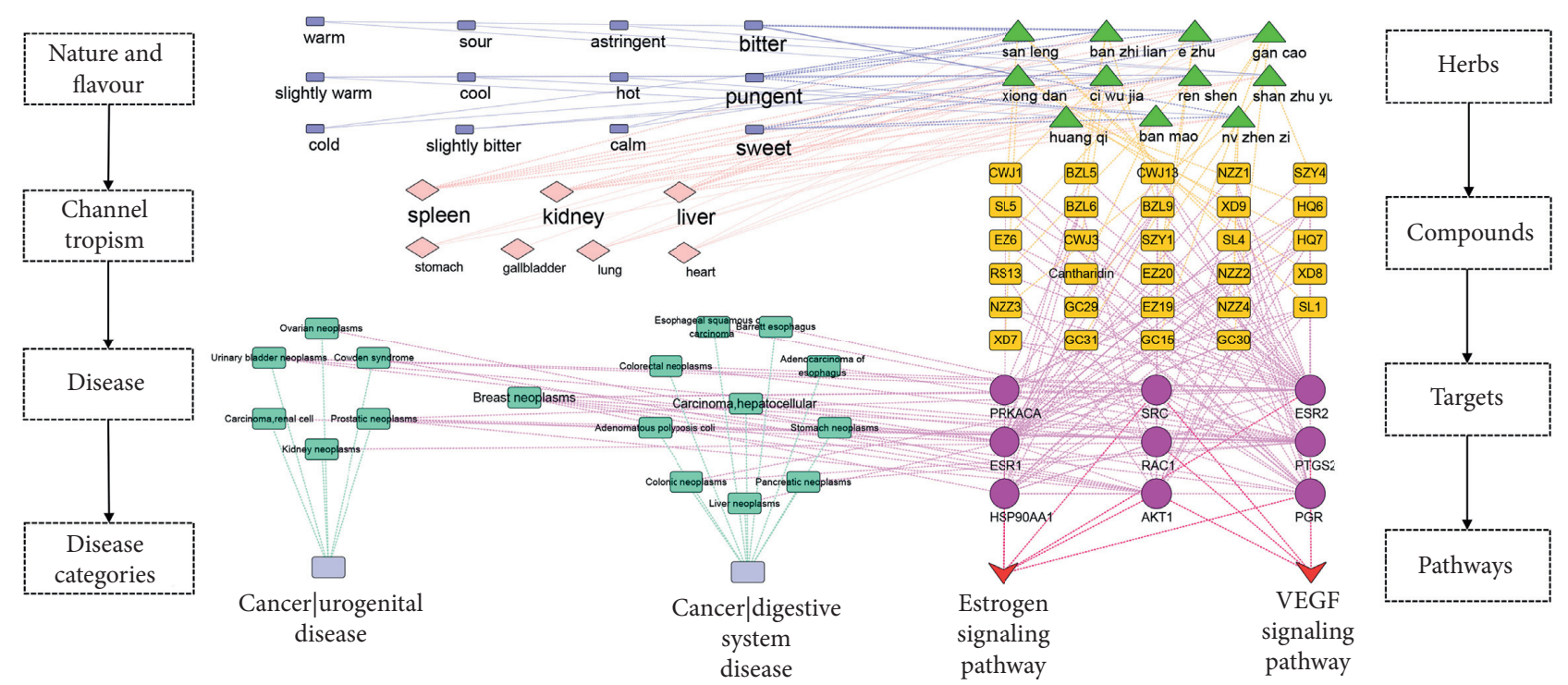

FIGURE 9: Multivariate-biological-network of compound banmao capsule (CBC). The left side stands for nature and flavor, channel tropism, and disease and disease categories, while the right side stands for herbs, compounds, targets, and pathways.

tumors, five urogenital tumors, and breast neoplasms (Figure 9).

To verify the accuracy of our prediction, 26 core compounds were examined by ADMET absorption level, suggesting that $65 \%$ of the compounds presented a good or moderate level (Figure 10(a)). Among them, $46 \%$ of the compounds showed a good level, indicating our prediction achieved great accuracy. Subsequently, 13 hub targets were docked with core compounds. The targets PTGS2 (-109.75), HSP90AA1 (-98.21), and AKT1 (-96.38) presented the most stable structures (Figure 10(b)). Gancaonin A (-108.53), quercetin (-108.80), scutellarin (-122.72), and sesamin 


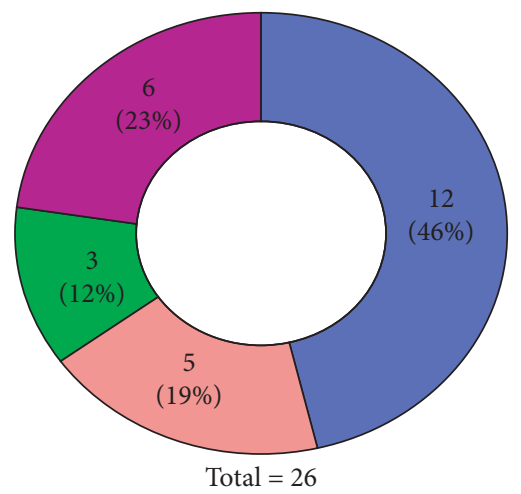

ADMET abosorption level

Good

Moderate

Very low

Not recorded in ETCM

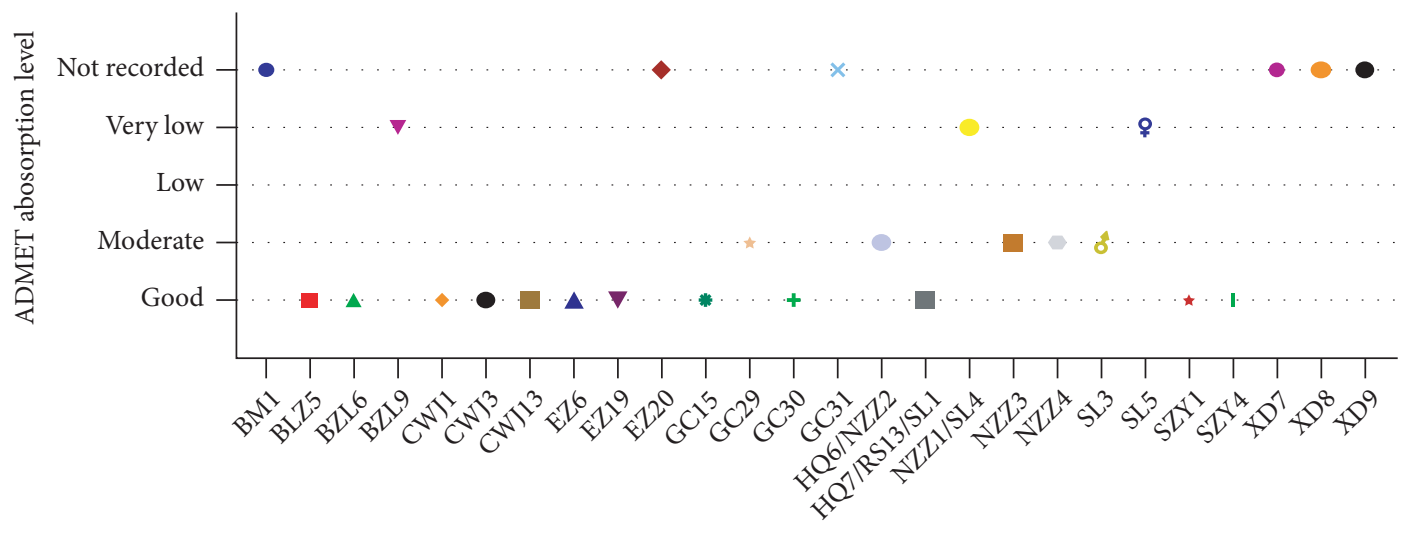

(a)

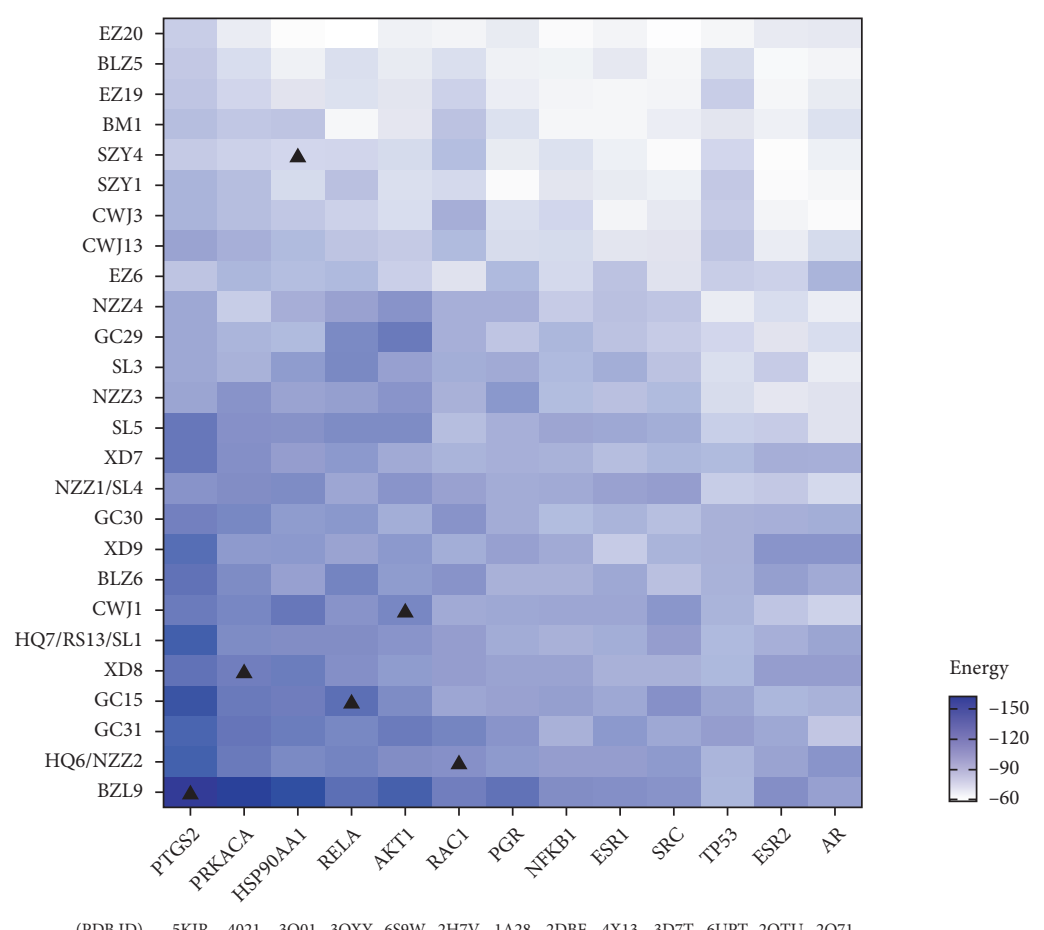

(b)

Figure 10: Continued. 


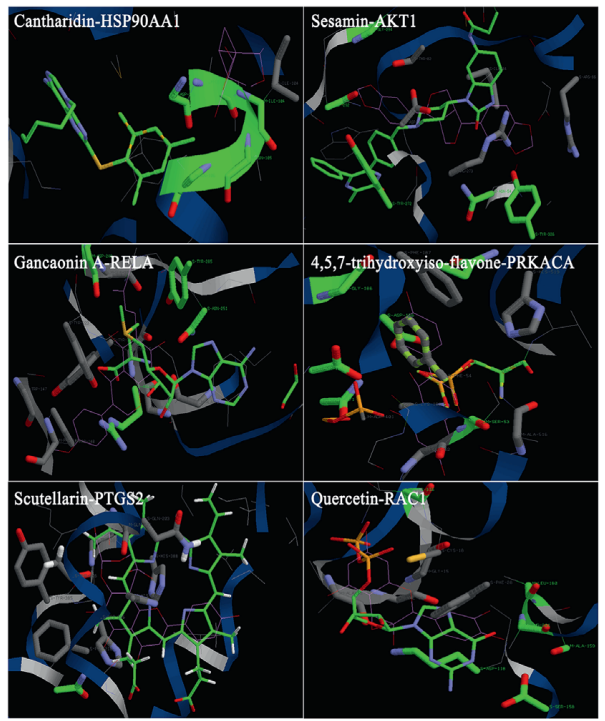

(c)
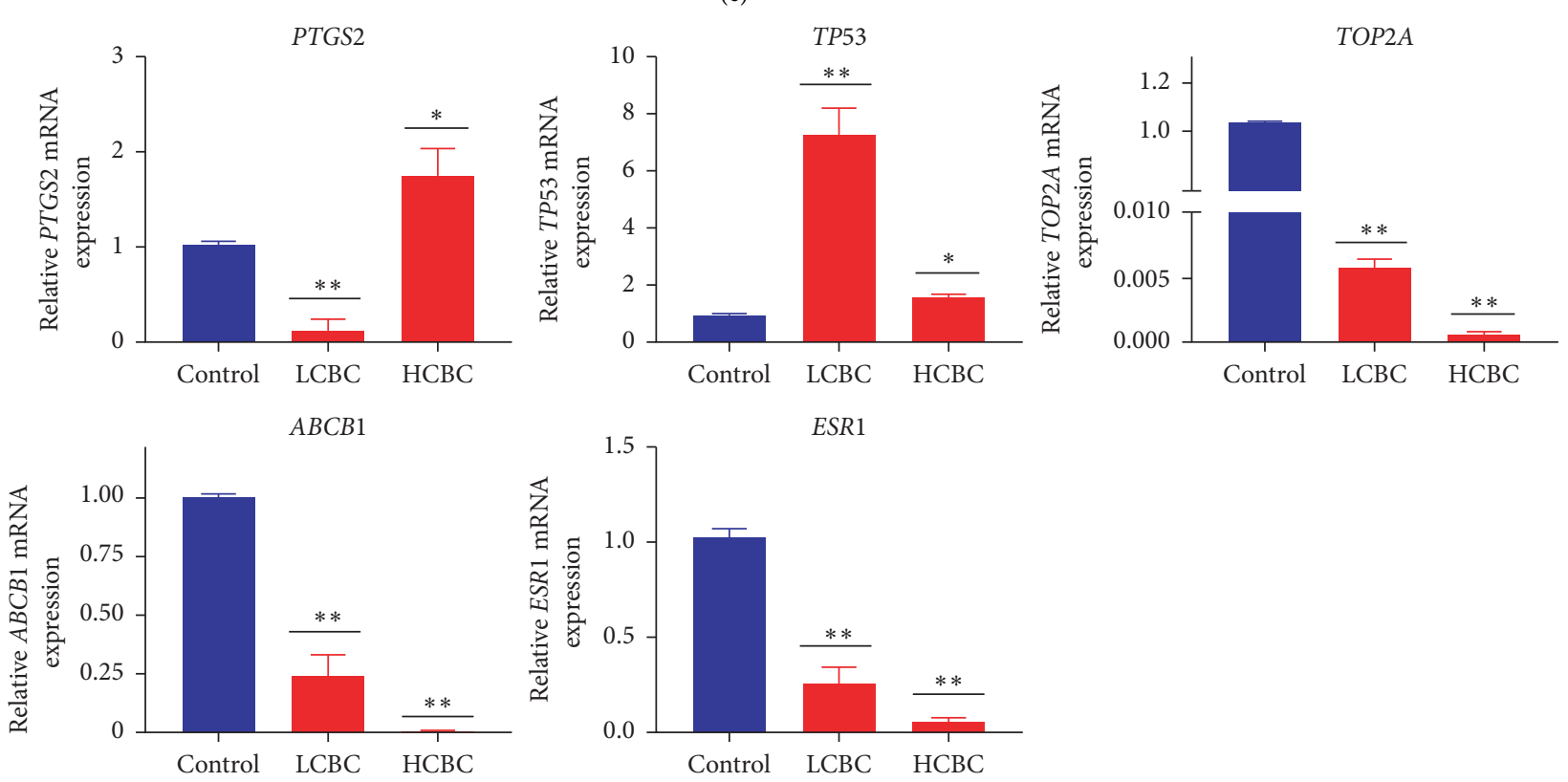

(d)

FIGURE 10: Results of predicted verification between compounds and targets in compound banmao capsule (CBC) against tumors. (a) Diagram of ADMET absorption level classification ratio of 26 key compounds of herbs from CBC. (b) Energy results in molecular docking. $X$ axis and $Y$ axis stand for gene targets and compounds. Colors varying from shallow to deep indicate that the docking accuracy is increasing. $\Delta$ stands for displayed diagram in c. (c) Best pose of molecular docking in key compounds and targets. (d) Gene mRNA expression levels of top targets in hepatocellular carcinoma by quantitative real-time PCR (qPCR) of CBC in SMMC-7721 cells. Compared with control, * presents $P<0.05$ and ${ }^{* *}$ presents $P<1$.

$(-102.37)$ showed a great potential against tumor targets by molecular docking and may be the main effective material basis of CBC. The ADMET absorption level of scutellarin was very low, but it still showed a great stable structure (Figure 10(c)).

3.1.7. Results of $q P C R$ for Liver Tumor Target Genes. The top five target genes PTGS2, TP53, ESR1, ABCB1, and TOP2A with a high degree of connectivity were identified in hepatocellular carcinoma of the antitumor location network. The effect of CBC on the expression of mRNA in SMMC7721 cells was explored by qPCR (Figure 10(d)). In this study, the mRNA expression levels of PTGS2, TOP2A, ESR1, and $A B C B 1$ were decreased in the LCBC group $(P<0.01)$, while PTGS2 was increased in the HCBC group $(P<0.05)$, and TP53 showed to be significantly increased in both doses $(P<0.01)$. Our results indicate that PTGS2, TP53, ESR1, 
$A B C B 1$, and $T O P 2 A$ may be the potential targets of $C B C$ in the treatment of liver tumors.

\section{Discussion}

$\mathrm{CBC}$ is a traditional antitumor drug of Miao ethnic in Guizhou Province of China, although their effective material basis and mechanisms of action remain unclear [37]. In this study, CBC's bioactive compounds and antitumor targets were identified. A PPI network was constructed to calculate hub targets, which were enriched to explore potential synergistic effects of CBC's components. Finally, key compounds and targets were validated using molecular docking and $\mathrm{qPCR}$.

CBC is clinically used as blood-activating, stasis-resolving, and detoxicating in TCM. Based on the Pharmacopoeia of the People's Republic of China, the 11 herbs of $\mathrm{CBC}$ were categorized according to the five properties and five flavors, reflecting the body's effect of "inclination" of the ups and downs of Yin and Yang and the "semi-interior phase" change during drug intervention [38]. To further explore the drug efficacy of complex compounds, nature and flavour analysis was applied. Our results indicated that CBC belonged to the pungent, bitter, and sweet categories. Pungent compounds have the potential action of "bloodactivating" and "stasis-resolving" and may have potential in treating liver diseases [39]. Herbs with bitter taste can be used for "discharging" and "down-bearing," referred to as "heat-clearing" and "detoxicating" herbs. Herbs with sweet taste may be used for "supplementation, moderation, and harmonization," referred to as "tonifying and replenishing" herbs [40]. However, sweet-tasting herbs with "spleenstrengthening" functions are used more frequently than herbs with a bitter taste for "clearing heat." There is a close relationship between recurrence and metastasis of breast cancer and liver. Herbs for "nourishing the yin-blood," "emolliating and soothing the liver," and "smoothing the meridians" are key in the treatment of breast cancer [40]. Our results indicated that $\mathrm{CBC}$ may have a multiaction "blood-activating," "stasis-resolving," "heat-clearing," and "detoxicating," and thus, it is potentially useful against breast and liver cancers.

To further explore the synergistic effects of CBC, the identification of the effective material basis and molecular mechanisms of CBC against tumors was analyzed.

4.1. Effective Material Basis of CBC. Twenty-six active compounds from 11 herbs were filtered as material basis of CBC by the herb-compound-target-pathway network analysis. In addition, the core compounds were verified by ADMET absorption level. Our results indicated that $65 \%$ of the core compounds showed a great absorption level, suggesting that our previous prediction was accurate. Among them, CTD, the core material basis of CBC and the most critical component in banmao, can efficiently inhibit the activity of mammalian and plant protein phosphatase $2 \mathrm{~A}$ (PP2A) in the treatment of tumors [41], and CTD may elevate leukocyte and enhance immunity [42].
Similarly, other compounds with great absorption levels, such as baicalein, sesamin, quercetin, and kaempferol, indicated a better interaction with hub targets by molecular docking. Besides, scutellarin may positively interact with the studied targets. Flavonoids of BZL, including scutellarin and baicalein from $\mathrm{CBC}$, have been found to significantly treat liver tumors $[43,44]$. The antitumor effects of sesamin have been mainly attributed to apoptotic, inflammatory, metastatic, and autophagocytic activities [45, 46]. Quercetin exhibits anticancer properties, especially in cancers located in the digestive and urogenital systems [47, 48]. Kaempferol was able to treat various tumors in the liver, skin, and colon through apoptosis, cell cycle arrest, and epithelial-mesenchymal transition [49]. In summary, baicalein, scutellarin, sesamin, quercetin, and kaempferol may be the core components of $\mathrm{CBC}$, which could influence the apoptotic, inflammatory, metastatic, and autophagic activity in tumor cells.

4.2. Antitumor Location of CBC and qPCR Verification of Liver Tumor Targets. The "channel tropism" is the core characteristic of TCM herbal property theory, which classifies drugs acting in the "body's viscera and meridians" to illustrate the selectivity of the drug's effects on a certain part of the body, thereby providing a basis for clinical dialectical medication [50]. Our results indicated that CBC belonged to "liver-meridian" and "kidney-meridian," confirming its indications against tumors in the digestive and urogenital systems, based on TCM theories. Based on the antitumor location network analysis of $\mathrm{CBC}$, digestive and urogenital systems tumors were mainly enriched in disease categories, especially in liver and breast cancer in disease terms.

The hub targets associated with hepatocellular carcinoma in antitumor location network of CBC were further verified by qPCR. The mRNA expression of PTGS2, TP53, TOP2A, ESR1, and $A B C B 1$ was significantly changed by $\mathrm{CBC}$ in SMMC-7721 cells, suggesting that these hub targets were crucial for the activity of $\mathrm{CBC}$ on hepatocellular carcinoma. The mRNA expression level of PTGS2 indicated a contrary trend in both doses, suggesting a dynamic regulation in the treatment of tumors with CBC.

The mechanistic studies implicate PTGS2 is overexpressed in liver cancer for promoting angiogenesis and inflammation [51]. Studies have shown that EZ from CBC was able to inhibit hepatocellular carcinoma by inhibiting PTGS2 in SMMC-7721 cell [52]. TP53, a tumor suppressor gene, mediates DNA repair, apoptosis, cell cycle arrest, autophagy, and metabolic processes [53]. TP53 can be both an activator or inhibitor of autophagy [54]. Studies have shown that CTD may treat tumors by upregulating TP53 [55]. CTD was also able to inhibit breast cancer cells by suppressing autophagy and inducing apoptosis [56]. In addition, ESR1 may play a role in the development and prognosis of liver cancer [57]. The ABCB1 are considered to be a prime factor for inducting multidrug resistance in liver cancer treatment [58]. However, the effect of CBC on ESR1, TOP2A, and ABCB1 remains unclear. In this study, we 
concluded that $\mathrm{CBC}$ may dynamically regulate PTGS2, TP53, TOP2A, ESR1, and ABCB1 targets by inhibiting SMMC-7721 cells.

4.3. Integrative Biological Network Analysis. The herbcompound-target-pathway network indicated that potential mechanisms of CBC involved 13 targets and five pathways, including VEGF signaling pathway, estrogen signaling pathway, and three tumor-associated pathways. Of these 13 targets, AKT1, SRC, RAC1, and PTGS2 are involved in VEGF signaling pathway. VEGF can increase angiogenesis and vascular permeability, contributing to key aspects of tumorigenesis, including the function of cancer cells and tumor initiation [59]. More importantly, several herbs or compounds were able to inhibit the VEGF signaling pathway against tumors. CTD inhibited angiogenesis by downregulating VEGF in vivo and vitro [60], whereas astragaloside IV of HQ from CBC treated a glioma by inhibiting VEGF [61]. SZY from CBC treated osteosarcoma by downregulation of VEGF [62], and EZ and HQ from CBC activity against lung tumors were based on the inhibition of VEGF [63]. Our results indicated that the VEGF signaling pathway may be a critical pathway of the CBC's inhibition of angiogenesis.

AKT1, also known as protein kinase B, activates the PI3K/AKT pathway during tumor growth [64]. Studies have shown that targeting AKT1 may impair angiogenesis [65]. $\mathrm{BM}$ and EZ from CBC may inhibit AKT1 [66, 67]. In addition, increased SRC target activity was identified in several malignancies [68]. Activation of SRC may further stimulate PI3K/Akt pathway. Studies have shown that CTD and SZY from CBC may inhibit SRC in vivo and vitro $[69,70]$, but the regulation of SRC of CBC still remains unclear. Furthermore, the overexpression of PTGS2 was able to activate VEGF for tumor growth and diffusion [71], and EZ from CBC could treat liver cancer by inhibiting PTGS2 and VEGF [52]. Thus, PTGS2 may be a potential target of CBC.

Of 13 targets, AKT1, ESR1, ESR2, HSP90AA1, PGR, PRKACA, and SRC are involved in estrogen signaling pathway, and AKT1 and SRC both involved in both VEGF and estrogen signaling pathways. Sustained estrogen exposure or deregulated estrogen signaling is associated with various types of tumor, including breast, endometrium, and prostate. Estrogen is a potential mediator of immunosuppression through modulation of protumors [72]. Studies have shown that san leng from $\mathrm{CBC}$ may treat breast cancer by regulating the estrogen receptor [73]. EZ from CBC may treat breast cancer through the inhibition of estrogenic activity $[74,75]$.

The estrogen receptor signaling is mediated by estrogen receptors, ESR1 and ESR2, while the majority of breast cancers have disregulated estrogen receptor signaling [76]. ESR1 is able to mediate the biological effects of the steroid hormone estrogen, while its mutation in breast cancer is a common mechanism of hormonal therapy resistance [77]. With the inhibition of ESR1, the risk of hormone breast cancer would be diminished [78]. ESR2 has been used as a prognostic molecular biomarker [79].

In this study, effective material basis, antitumor location, and mechanism of $\mathrm{CBC}$ were integrated in a multivariate biological network (Figure 9), which combined TCM theories and molecular biological network, identifying the multicompound-target-pathway of CBC against tumors. Our results indicated that $\mathrm{CBC}$ displays is "blood-activating," "stasis-resolving," "heat-clearing," and "detoxicating," and this is associated with its pharmacological function in VEGF, estrogen signaling pathway, PTGS2, and ESR1. These targets were further verified by qPCR. Furthermore, CBC's activity as antitumor against liver and breast tumors was confirmed by a literature review.

\section{Conclusion}

Effective material basis and potential molecular mechanisms of CBC against tumors have been here explored, based on TCM theories and biological network analysis. These were further verified by qPCR and molecular docking. The potential therapeutic location and hub targets of CBC were identified by multivariate biological network integration. Future studies should focus on CBC's mechanisms of action by molecular experiments in vivo and in vitro. Additionally, these experiments would further validate our results as per the inherent limitations of network predictions.

\section{Data Availability}

The data used to support the findings of this study are included within the article.

\section{Conflicts of Interest}

The authors declare that they have no conflicts of interest.

\section{Acknowledgments}

This research was supported by the National Natural Science Foundation of China (Grant nos. 81760746 and 81803838), Science and Technology Department of Guizhou Province of China (Grant nos. [2016] 2834, LH [2015] 7532, [2020] 1Y376, and [2019] 1346), Science and Technology Department of Zunyi City of Zuniyi City of Guizhou Province of China (Grant nos. [2016] 35 and [2016] 33), Innovation Talent Team of Guizhou Science and Technology Department (qiankehe platform talents, Grant no. [2020] 5007), and Education Department of Guizhou Province of China (GNYL, Grant nos. [2017] 006, [2017] 078, and [2012] 311).

\section{Supplementary Materials}

Table 1: detail compounds in each herb from compound banmao capsule (CBC). (Supplementary Materials)

\section{References}

[1] C. Allemani, T. Matsuda, V. Di Carlo et al., "Global surveillance of trends in cancer survival 2000-14 (CONCORD3): analysis of individual records for 37513025 patients diagnosed with one of 18 cancers from 322 population-based registries in 71 countries," The Lancet, vol. 391, no. 10125, pp. 1023-1075, 2018. 
[2] D. Anwanwan, S. K. Singh, S. Singh, V. Saikam, and R. Singh, "Challenges in liver cancer and possible treatment approaches," Biochimica et Biophysica Acta - Reviews on Cancer, vol. 1873, no. 1, p. 188314, 2020.

[3] J. Zhou, T. Zhou, M. Chen et al., "Research progress on synergistic anti-tumor mechanisms of compounds in traditional Chinese medicine," Journal of Traditional Chinese Medicine, vol. 34, no. 1, pp. 100-105, 2014.

[4] S. Wang, S. Long, and W. Wu, "Application of traditional Chinese medicines as personalized therapy in human cancers," The American Journal of Chinese Medicine, vol. 46, no. 05, pp. 953-970, 2018.

[5] X. Liu, M. Li, X. Wang et al., "Effects of adjuvant traditional Chinese medicine therapy on long-term survival in patients with hepatocellular carcinoma," Phytomedicine, vol. 62, p. 152930, 2019.

[6] Y. Y. Cao, M. Wang, K. S. Huang, Q. Yang, and M. Ding, "Proteomic analysis of differentially expressed proteins in human hepatoma SMMC-7721 cells induced by Fufang Banmao capsule serum," Zhongguo Zhong Yao Za Zhi, vol. 32, no. 9, pp. 831-834, 2007.

[7] J. J. Han, J. M. Yu, H. Y. Wu, J. B. Liu, B. Song, and D. W. Xue, "Inhibitory effect of compound cantharides capsule on the proliferation of xenografts of human hepatocellular carcinoma HepG (2) 215 in mice," Zhonghua Zhong Liu Za Zhi, vol. 34, no. 11, pp. 821-825, 2012.

[8] Y. Sun, D. Zhang, M. Mao, Y. Lu, and N. Jiao, "Roles of p38 and JNK protein kinase pathways activated by compound cantharidin capsules containing serum on proliferation inhibition and apoptosis of human gastric cancer cell line," Experimental and Therapeutic Medicine, vol. 14, no. 2, pp. 1809-1817, 2017.

[9] P. Poornima, J. D. Kumar, Q. Zhao, M. Blunder, and T. Efferth, "Network pharmacology of cancer: from understanding of complex interactomes to the design of multitarget specific therapeutics from nature," Pharmacological Research, vol. 111, pp. 290-302, 2016.

[10] M. Kibble, N. Saarinen, J. Tang, K. Wennerberg, S. Mäkelä, and T. Aittokallio, "Network pharmacology applications to map the unexplored target space and therapeutic potential of natural products," Natural Product Reports, vol. 32, no. 8, pp. 1249-1266, 2015.

[11] H.-Y. Xu, Y.-Q. Zhang, Z.-M. Liu et al., "ETCM: an encyclopaedia of traditional Chinese medicine," Nucleic Acids Research, vol. 47, no. D1, pp. D976-D982, 2019.

[12] G. R. Bickerton, G. V. Paolini, J. Besnard, S. Muresan, and A. L. Hopkins, "Quantifying the chemical beauty of drugs," Nature Chemistry, vol. 4, no. 2, pp. 90-98, 2012.

[13] Z.-J. Yao, J. Dong, Y.-J. Che et al., "TargetNet: a web service for predicting potential drug-target interaction profiling via multi-target SAR models," Journal of Computer-Aided Molecular Design, vol. 30, no. 5, pp. 413-424, 2016.

[14] G. Stelzer, N. Rosen, I. Plaschkes et al., "The GeneCards suite: from gene data mining to disease genome sequence analyses," Current Protocols in Bioinformatics, vol. 54, pp. 1-31 30 33, 2016.

[15] D. Szklarczyk, A. L. Gable, D. Lyon et al., "STRING v11: protein-protein association networks with increased coverage, supporting functional discovery in genome-wide experimental datasets," Nucleic Acids Research, vol. 47, no. D1, pp. D607-D613, 2019.

[16] G. Min lianY. Yang et al., "Discussion on the application of "four properties and five tastes" in breast cancer," Liaoning Journal of Traditional Chinese Medicine, 2021.
[17] M. V. Kuleshov, M. R. Jones, A. D. Rouillard et al., "Enrichr: a comprehensive gene set enrichment analysis web server 2016 update," Nucleic Acids Research, vol. 44, no. W1, pp. W90-W97, 2016.

[18] Z. R. Su JinF. Guo et al., "Molecular mechanism of Shuguan Granules in treating angina based on integrative pharmacology," Zhong Guo Zhong Yao Za Zhi, vol. 44, no. 7, pp. 1425-1435, 2019.

[19] J.-M. Yang and C.-C. Chen, "GEMDOCK: a generic evolutionary method for molecular docking," Proteins: Structure, Function, and Bioinformatics, vol. 55, no. 2, pp. 288-304, 2004.

[20] W. Jiang, W. Li, L. Han et al., "Biologically active triterpenoid saponins fromAcanthopanax senticosus," Journal of Natural Products, vol. 69, no. 11, pp. 1577-1581, 2006.

[21] T. Gong, C. F. Wang, J. R. Yuan et al., "Inhibition of tumor growth and immunomodulatory effects of flavonoids and scutebarbatines of scutellaria barbata D. Don in lewis-bearing C57bl/6 mice," Evidence-Based Complementary and Alternative Medicine, vol. 2015, p. 630760, 2015.

[22] Y. Jung, U. Jerng, and S. Lee, "A systematic review of anticancer effects of radix astragali," Chinese Journal of Integrative Medicine, vol. 22, no. 3, pp. 225-236, 2016.

[23] J. H. Kim, Y.-S. Yi, M.-Y. Kim, and J. Y. Cho, "Role of ginsenosides, the main active components of Panax ginseng, in inflammatory responses and diseases," Journal of Ginseng Research, vol. 41, no. 4, pp. 435-443, 2017.

[24] L. Žiberna, D. Šamec, A. Mocan et al., "Oleanolic acid alters multiple cell signaling pathways: implication in cancer prevention and therapy," International Journal of Molecular Sciences, vol. 18, no. 3, 2017.

[25] Y. Dong, Z. L. Feng, H. B. Chen, F. S. Wang, and J. H. Lu, "Corni Fructus: a review of chemical constituents and pharmacological activities," Chinese Medicine, vol. 13, p. 34, 2018.

[26] G. Wang, J. Dong, and L. Deng, "Overview of cantharidin and its analogues," Current Medicinal Chemistry, vol. 25, no. 17, pp. 2034-2044, 2018.

[27] J. Yan, F.-R. Wang, and F.-J. He, “The mechanism analysis of the tri - rhizoma drug in Diaomozhibeng cream based on the network pharmacology," Lishizhen Medicine and Materia Medica Research, vol. 29, no. 2, pp. 489-491, 2018.

[28] J. Zheng, M. Wu, H. Wang et al., "Network pharmacology to unveil the biological basis of health-strengthening herbal medicine in cancer treatment," Cancers (Basel), vol. 1011 pages, 2018.

[29] Z.-J Zhuang, R. Li, and H. Ci-hui, "Molecular mechanism of Ligustri Lucidi fructus-astragali radix for anti-cancer based on network pharmacology," Chinese Journal of Experimental Traditional Medical Formulae, vol. 25, no. 12, pp. 195-202, 2019.

[30] K. M. Lau, G. G. Yue, Y. Y. Chan et al., "A review on the immunomodulatory activity of Acanthopanax senticosus and its active components," Chinese Medicine, vol. 14, p. 25, 2019.

[31] N. Li, T. Zhou, F. Wu et al., "Pharmacokinetic mechanisms underlying the detoxification effect of Glycyrrhizae Radix et Rhizoma (Gancao): drug metabolizing enzymes, transporters, and beyond," Expert Opinion on Drug Metabolism \& Toxicology, vol. 15, no. 2, pp. 167-177, 2019.

[32] J. Liu, S. H. Nile, G. Xu, Y. Wang, and G. Kai, "Systematic exploration of Astragalus membranaceus and Panax ginseng as immune regulators: insights from the comparative biological and computational analysis," Phytomedicine, vol. 2019, Article ID 153077, 2019. 
[33] A. Nair, A. Amalraj, J. Jacob, A. B. Kunnumakkara, and S. Gopi, "Non-curcuminoids from turmeric and their potential in cancer therapy and anticancer drug delivery formulations," Biomolecules, vol. 9, no. 1, 2019.

[34] B. Zhai, N. Zhang, X. Han et al., "Molecular targets of betaelemene, a herbal extract used in traditional Chinese medicine, and its potential role in cancer therapy: a review," Biomed Pharmacother, vol. 114, Article ID 108812, 2019.

[35] B. H. H. Guangzhao, "Determination of four active components in compound banmao capsules by HPLC," China Pharmaceuticals, vol. 29, no. 9, 2020.

[36] H. C. L. WangF. Li et al., "Effect and mechanism of Fufang Xiongdan Diyanye in the treatment of conjunctivitis based on network pharmacology," Journal of Guangdong Pharmaceutical University, vol. 36, no. 1, pp. 78-83, 2020.

[37] Y. Liu, Y. Li, X. Wang et al., "Fufang banmao capsule, a traditional Chinese medicinal formulation, enhances the survival of patients with hepatocellular carcinoma and vp3-4 portal vein tumor thrombosis undergoing supportive treatment," The Journal of Alternative and Complementary Medicine, vol. 26, no. 10, pp. 956-965, 2020.

[38] R. Fu, J. Li, H. Yu, Y. Zhang, Z. Xu, and C. Martin, "The yin and Yang of traditional Chinese and western medicine," Medicinal Research Reviews, vol. 2021, 2021.

[39] Z. Chen, Y. Cao, Y. Zhang, and Y. Qiao, "A novel discovery: holistic efficacy at the special organ level of pungent flavored compounds from pungent traditional Chinese medicine," International Journal of Molecular Sciences, vol. 20, no. 3, 2019.

[40] Y. He, X. Zheng, C. Sit et al., "Using association rules mining to explore pattern of Chinese medicinal formulae (prescription) in treating and preventing breast cancer recurrence and metastasis," Journal of Translational Medicine, vol. 10, no. Suppl 1, p. S12, 2012.

[41] A. McCluskey, C. Walkom, M. C. Bowyer, S. P. Ackland, E. Gardiner, and J. A. Sakoff, "Cantharimides: a new class of modified cantharidin analogues inhibiting protein phosphatases 1 and 2A," Bioorganic \& Medicinal Chemistry Letters, vol. 11, no. 22, pp. 2941-2946, 2001.

[42] G.-S. Wang, "Medical uses of mylabris in ancient China and recent studies," Journal of Ethnopharmacology, vol. 26, no. 2, pp. 147-162, 1989.

[43] Z.-J. Dai, B.-F. Wang, W.-F. Lu et al., "Total flavonoids of Scutellaria barbata inhibit invasion of hepatocarcinoma via MMP/TIMP in vitro," Molecules, vol. 18, no. 1, pp. 934-950, 2013.

[44] B. Bie, J. Sun, Y. Guo et al., "Baicalein: a review of its anticancer effects and mechanisms in Hepatocellular Carcinoma," Biomedicine \& Pharmacotherapy, vol. 93, pp. 1285-1291, 2017.

[45] A. F. Majdalawieh, M. Massri, and G. K. Nasrallah, "A comprehensive review on the anti-cancer properties and mechanisms of action of sesamin, a lignan in sesame seeds (Sesamum indicum)," European Journal of Pharmacology, vol. 815, pp. 512-521, 2017.

[46] H. Dou, S. Yang, Y. Hu, D. Xu, L. Liu, and X. Li, "Sesamin induces ER stress-mediated apoptosis and activates autophagy in cervical cancer cells," Life Sciences, vol. 200, pp. 87-93, 2018.

[47] M. Hashemzaei, A. D. Far, A. Yari et al., "Anticancer and apoptosis-inducing effects of quercetin in vitro and in vivo," Oncology Reports, vol. 38, no. 2, pp. 819-828, 2017.

[48] K. Hu, L. Miao, T. J. Goodwin, J. Li, Q. Liu, and L. Huang, "Quercetin remodels the tumor microenvironment to improve the permeation, retention, and antitumor effects of nanoparticles," ACS Nano, vol. 11, no. 5, pp. 4916-4925, 2017.

[49] M. Imran, B. Salehi, J. Sharifi-Rad et al., "Kaempferol: a key emphasis to its anticancer potential," Molecules, vol. 24, no. 12, 2019.

[50] P. Liu, S. Liu, G. Chen, and P. Wang, "Understanding channel tropism in traditional Chinese medicine in the context of systems biology," Frontiers of Medicine, vol. 7, no. 3, pp. 277-279, 2013.

[51] P. Anand, P. Filipenko, J. Huaman et al., "Selective inhibition of liver cancer cells using venom peptide," Marine Drugs, vol. 1710 pages, 2019.

[52] N. Li, D. Zheng, J. Xue et al., "Cidan inhibits liver cancer cell growth by reducing COX-2 and VEGF expression and cell cycle arrest," Experimental and Therapeutic Medicine, vol. 9, no. 5, pp. 1709-1718, 2015.

[53] B. J. Aubrey, A. Strasser, and G. L. Kelly, "Tumor-suppressor functions of the TP53 pathway," Cold Spring Harbor Perspectives in Medicine, vol. 6, no. 5, 2016.

[54] P. Mosaddeghi, M. Eslami, M. Farahmandnejad et al., "A systems pharmacology approach to identify the autophagyinducing effects of Traditional Persian medicinal plants," Scientific Reports, vol. 11, no. 1, p. 336, 2021.

[55] X. Tian, G. Zeng, X. Li, Z. Wu, and L. Wang, "Cantharidin inhibits cell proliferation and promotes apoptosis in tongue squamous cell carcinoma through suppression of miR-214 and regulation of p53 and Bcl-2/Bax," Oncology Reports, vol. 33, no. 6, pp. 3061-3068, 2015.

[56] H.-C. Li, Z.-H. Xia, Y.-F. Chen et al., "Cantharidin inhibits the growth of triple-negative breast cancer cells by suppressing autophagy and inducing apoptosis in vitro and in vivo," Cellular Physiology and Biochemistry, vol. 43, no. 5, pp. 1829-1840, 2017.

[57] H. Sun, J. Hou, W. Shi, and L. Zhang, "Estrogen receptor 1 (ESR1) genetic variations in cancer risk: a systematic review and meta-analysis," Clinics and Research in Hepatology and Gastroenterology, vol. 39, no. 1, pp. 127-135, 2015.

[58] A. Kumar and V. Jaitak, "Natural products as multidrug resistance modulators in cancer," European Journal of Medicinal Chemistry, vol. 176, pp. 268-291, 2019.

[59] H. L. Goel and A. M. Mercurio, "VEGF targets the tumour cell," Nature Reviews Cancer, vol. 13, no. 12, pp. 871-882, 2013.

[60] T. Wang, J. Liu, and X.-Q. Xiao, "Cantharidin inhibits angiogenesis by suppressing VEGF-induced JAK1/STAT3, ERK and AKT signaling pathways," Archives of Pharmacal Research, vol. 38, no. 2, pp. 282-289, 2015.

[61] B. Li, F. Wang, N. Liu, W. Shen, and T. Huang, "Astragaloside IV inhibits progression of glioma via blocking MAPK/ERK signaling pathway," Biochemical and Biophysical Research Communications, vol. 491, no. 1, pp. 98-103, 2017.

[62] C.-L. Liao, J.-H. Lin, J.-C. Lien et al., "The crude extract ofCorni Fructusinhibits the migration and invasion of U-2 OS human osteosarcoma cells through the inhibition of matrix metalloproteinase-2/-9 by MAPK signaling," Environmental Toxicology, vol. 30, no. 1, pp. 53-63, 2015.

[63] C. Xu, Y. Wang, J. Feng, R. Xu, and Y. Dou, "Extracts from Huangqi (Radix Astragali Mongoliciplus) and Ezhu (Rhizoma Curcumae Phaeocaulis) inhibit Lewis lung carcinoma cell growth in a xenograft mouse model by impairing mitogenactivated protein kinase signaling, vascular endothelial growth factor production, and angiogenesis," Journal of Traditional Chinese Medicine, vol. 39, no. 4, pp. 559-565, 2019. 
[64] O. P. Martínez-Rodríguez, M. D. R. Thompson-Bonilla, and M. E. Jaramillo-Flores, "Association between obesity and breast cancer: molecular bases and the effect of flavonoids in signaling pathways," Critical Reviews in Food Science and Nutrition, vol. 202023 pages, 2020.

[65] A. Alwhaibi, A. Verma, M. S. Adil, and P. R. Somanath, "The unconventional role of Akt1 in the advanced cancers and in diabetes-promoted carcinogenesis," Pharmacological Research, vol. 145, Article ID 104270, 2019.

[66] G. Zhao, X. Han, S. Zheng et al., "Curcumin induces autophagy, inhibits proliferation and invasion by downregulating AKT/mTOR signaling pathway in human melanoma cells," Oncology Reports, vol. 35, no. 2, pp. 1065-1074, 2016.

[67] M. Song, X. Wang, Y. Luo et al., "Cantharidin suppresses gastric cancer cell migration/invasion by inhibiting the PI3K/ Akt signaling pathway via CCAT1," Chem Biol Interact, vol. 317, Article ID 108939, 2020.

[68] H. Krishnan, W. T. Miller, F. J. Blanco, and G. S. Goldberg, "Src and podoplanin forge a path to destruction," Drug Discovery Today, vol. 24, no. 1, pp. 241-249, 2019.

[69] X. Hu, X. Wu, J. Xu, J. Zhou, X. Han, and J. Guo, "Src kinase up-regulates the ERK cascade through inactivation of protein phosphatase 2A following cerebral ischemia," BMC Neuroscience, vol. 10, no. 1, p. 74, 2009.

[70] C.-C. Yang, X.-X. Kuai, W.-B. Gao et al., "Morroniside-Induced PP2A activation antagonizes tau hyperphosphorylation in a cellular model of neurodegeneration," Journal of Alzheimer's Disease, vol. 51, no. 1, pp. 33-44, 2016.

[71] X.-S. Meng, N.-N. Li, Y.-R. Bao, S. Wang, and T.-J. Li, "Evidence for the involvement of COX-2/VEGF and PTEN/ $\mathrm{Pl} 3 \mathrm{~K} / \mathrm{AKT}$ pathway the mechanism of oroxin B treated liver cancer," Pharmacognosy Magazine, vol. 14, no. 54, 2018.

[72] N. J. Rothenberger, A. Somasundaram, and L. P. Stabile, "The role of the estrogen pathway in the tumor microenvironment," International Journal of Molecular Sciences, vol. 19, no. 2, 2018.

[73] Y.-Z. Wu, J. Sun, and Y.-B. Wang, "Selective estrogen receptor modulator: a novel polysaccharide from Sparganii Rhizoma induces apoptosis in breast cancer cells," Carbohydrate Polymers, vol. 163, pp. 199-207, 2017.

[74] Y.-W. Li, G.-Y. Zhu, X.-L. Shen, J.-H. Chu, Z.-L. Yu, and W.-F. Fong, "Furanodienone inhibits cell proliferation and survival by suppressing ER $\alpha$ signaling in human breast cancer MCF-7 cells," Journal of Cellular Biochemistry, vol. 112, no. 1, pp. 217-224, 2011.

[75] M.-S. Lim, S.-Y. Choung, and K. W. Jeong, "Germacrone inhibits estrogen receptor $\alpha$-mediated transcription in MCF-7 breast cancer cells," Phytotherapy Research, vol. 30, no. 12, pp. 2036-2043, 2016.

[76] T. Saha, S. Makar, R. Swetha, G. Gutti, and S. K. Singh, "Estrogen signaling: an emanating therapeutic target for breast cancer treatment," European Journal of Medicinal Chemistry, vol. 177, pp. 116-143, 2019.

[77] D. Dustin, G. Gu, and S. A. W. Fuqua, "ESR1 mutations in breast cancer," Cancer, vol. 125, no. 21, pp. 3714-3728, 2019.

[78] T.-C. Hung, W.-Y. Lee, K.-B. Chen, Y.-C. Chan, and C. Y.-C. Chen, "Investigation of estrogen receptor (ESR1) for breast cancer from traditional Chinese medicine," BioMed Research International, vol. 2014, pp. 1-12, 2014.

[79] V. Duong, A. Licznar, R. Margueron et al., "ER $\alpha$ and ER $\beta$ expression and transcriptional activity are differentially regulated by HDAC inhibitors," Oncogene, vol. 25, no. 12, pp. 1799-1806, 2005. 